Sharif University of Technology
Scientia Iranica
SCIENTIA
IRAN I CA

\title{
A differential quadrature procedure for free vibration of rectangular plates involving free corners
}

\author{
S.A. Eftekhari ${ }^{\mathrm{a}, *}$ and A.A. Jafari ${ }^{\mathrm{b}}$ \\ a. Young Researchers and Elite Club, Karaj Branch, Islamic Azad University, Karaj, P.O. Box 31485-313, Iran. \\ b. Department of Mechanical Engineering, K. N. Toosi University of Technology, Tehran, P.O. Box 19395-1999, Iran. \\ Received 17 October 2014; received in revised form 21 July 215; accepted 15 September 2015
}

\section{KEYWORDS \\ Simple DQM \\ formulation; \\ Rectangular plates; \\ Boundary conditions; \\ Free edges; \\ Free Corners.}

\begin{abstract}
The Differential Quadrature Method (DQM) is one of the most powerful approximation methods for analyzing the free vibration of rectangular plates. It is easy to use and straightforward to implement. However, in spite of its many advantages, the conventional DQM has some limitations in determining the natural frequencies of rectangular plates involving free corners. This is because it is very difficult to implement the free corner boundary condition in conventional DQM. As a result, the method may exhibit some convergence problems and this may lead to erroneous and oscillatory results for natural frequencies of rectangular plates involving free corners. To overcome this difficulty, this paper presents a simple DQM formulation in which all the natural boundary conditions, including the free corner boundary condition, are implemented in an easy manner. Its accuracy and efficiency are demonstrated through the vibration analysis of rectangular plates with different combinations of free edges and free corners. Numerical results prove that the proposed method can produce much better accuracy than the conventional DQM while exhibiting a monotonic convergence behavior with respect to the number of sampling points. Furthermore, unlike the conventional DQM, solutions of the proposed method are not very sensitive to the sampling point distribution.

(C) 2016 Sharif University of Technology. All rights reserved.
\end{abstract}

\section{Introduction}

Rectangular plates are important structural components that are extensively used in various fields of engineering such as civil, mechanical, aerospace, marine, and structural engineering. Therefore, proper understanding of the vibration characteristics of such structural elements is crucial for the structural designers.

In general, there are two kinds of methods that can be used to solve the free vibration problem of rectangular plates, namely, the analytical and numerical

\footnotetext{
* Corresponding author. Tel.: +989194618599; Fax: +982634418156.

E-mail address: aboozar.eftekhari@gmail.com (S.A. Eftekhari)
}

methods. The analytical methods often provide better information about vibration characteristics of rectangular plates. But, their applications are limited to plate problems with simple boundary conditions such as Levy-type boundary conditions $[1,2]$. This limitation is caused by the complexities introduced by the satisfaction of the free edges and free corner boundary conditions. To overcome the limitations of the analytical methods, various approximate or numerical methods such as the Ritz method [3-6], the extended Kantorovich approach [7], the finite element method $[8,9]$, the BEM-based meshless method [10], the moving least squares differential quadrature method [11], semianalytic differential quadrature method [12], the finite difference method [13], the spectral element method [14], and the discrete singular convolution method $[15,16]$ have been developed by researchers 
to study the behavior of rectangular or other shaped plates with general boundary conditions.

Among the approximate methods used for solving the present problem, the Differential Quadrature Method (DQM) is one of the most convenient methods to obtain natural frequencies of rectangular plates [1720]. It is simple to use and also straightforward to implement. However, in spite of its many useful features, the conventional DQM has its own drawbacks in implementation in the differential equations with multiple boundary conditions at the boundary points, especially for fourth-order governing differential equations of classical beam and plate problems. For instance, in solving the vibration problem of rectangular plates with general boundary conditions, Malik and Bert [21] indicated that the solutions of conventional DQM for rectangular plates having adjacent free edges (or free corners) might not exhibit convergence trend and erroneous results might be obtained. To solve this difficulty, Shu and Du [22] proposed an approach referred to as the direct Coupling of Boundary Conditions with discrete Governing Equations (CBCGE) for implementing the general boundary conditions for the free vibration analysis of rectangular plates. The CBCGE approach was shown to work well for the rectangular plates without free corners, but it encountered some issues when applied to the rectangular plates involving free corners. In this case, the numerical solutions of the CBCGE approach were highly sensitive to the sampling point distribution. For instance, the solutions of the CBCGE approach with conventional non-uniform sampling points were quite erroneous. To overcome this difficulty, Shu and Du [22] proposed the use of stretched sampling points where the sampling points were stretched toward the plate boundaries. Although rather accurate solutions were obtained using the proposed stretched sampling points, the obtained solutions did not show a monotonic convergence with increasing number of sampling points and, in some cases, the natural frequencies were found to behave oscillatory.

It can be seen that a simple and general formulation based on the conventional DQM that can easily handle the plate problem with general boundary conditions is still missing. Therefore, this paper intends to present a simple and accurate DQM formulation in which all the natural boundary conditions, including the free corner boundary conditions, are satisfied in an easy and accurate manner. To demonstrate its accuracy and stability, the proposed methodology is applied to solve the vibration problem of rectangular plates with various combinations of free edges and free corners. Comparison of obtained results with those in recent literature shows that the proposed methodology is capable of producing highly accurate solutions while exhibiting a monotonic convergence behavior with respect to the number of DQM sampling points. Furthermore, the proposed approach can produce much better accuracy than the conventional DQM formulations for rectangular plates involving free corners.

\section{Differential quadrature method}

The DQM is a numerical solution technique for initial and/or boundary value problems [23]. It was first developed by Richard Bellman and his associates in the early 1970's [24,25]. Since its introduction, the DQM has been successfully applied to a variety of engineering problems [26-45]. Most of these applications are related to static and dynamic analyses of structural components like beams, plates, and shells. Newer applications include the use of DQM for solving moving load problems [46,47] and fluid-structure interaction problems $[48,49]$. The results of many research works show that the DQM is computationally efficient and is applicable to a large class of initial and/or boundary value problems. However, as we discussed in introduction, the implementation of multiple boundary conditions is not an easy task when applying the DQM to higher-order partial differential equations. To overcome this limitation, this paper is devoted to present a simple and accurate DQM formulation in which the multiple boundary conditions are implemented in an easy and simple manner.

The DQM is based on the idea that the derivative of a function with respect to a coordinate direction at any discrete point can be expressed by a weighted linear sum of the function values at all the discrete points chosen in that direction. For instance, the $r$ th-order $X$ derivative of the function $W(X, Y)$ at a sample point $\left(X_{i}, Y\right)$ can be expressed as [23]:

$$
\begin{aligned}
& \frac{\partial^{r} W\left(X_{i}, Y\right)}{\partial X^{r}}=\sum_{j=1}^{n} A_{i k}^{(r)} W\left(X_{k}, Y\right), \\
& i=1,2, \cdots, n,
\end{aligned}
$$

where $n$ is the number of sample points in the $X$-direction, and $A_{i k}^{(r)}$ is the $r$ th-order $X$-derivative weighting coefficient associated with the $X=X_{i}$ point.

It follows from Eq. (1) of which the quadrature rules may be written collectively in matrix form as:

$$
\frac{\partial^{r}}{\partial X^{r}}\{\mathbf{W}(Y)\}=\left[\mathbf{A}^{(\mathbf{r})}\right]\{\mathbf{W}(Y)\}
$$

where:

$$
\begin{aligned}
& \{\mathbf{W}(Y)\} \\
& \left.\quad=\left[W\left(X_{1}, Y\right) W\left(X_{2}, Y\right) \cdots W\left(X_{n}, Y\right)\right]^{T}\right],
\end{aligned}
$$




$$
\left[\mathbf{A}^{(r)}\right]=\left[\begin{array}{cccc}
A_{11}^{(r)} & A_{12}^{(r)} & \cdots & A_{1 n}^{(r)} \\
A_{21}^{(r)} & A_{22}^{(r)} & \cdots & A_{2 n}^{(r)} \\
\vdots & \vdots & \vdots & \vdots \\
A_{n 1}^{(r)} & A_{n 2}^{(r)} & \cdots & A_{n n}^{(r)}
\end{array}\right] .
$$

The weighting coefficients of the first-order derivative can be obtained from the following algebraic formulations [50]:

$$
A_{i k}^{(1)}= \begin{cases}\frac{\Pi\left(X_{i}\right)}{\left(X_{i}-X_{k}\right) \Pi\left(X_{k}\right)} \quad i \neq k, \quad i, k=1,2, \cdots, n \\ -\sum_{j=1, j \neq i}^{n} A_{i j}^{(1)} \quad i=k, \quad i=1,2, \cdots, n\end{cases}
$$

where $\Pi(X)$ is defined as:

$$
\Pi\left(X_{i}\right)=\prod_{j=1, j \neq i}^{n}\left(X_{i}-X_{j}\right)
$$

The weighting coefficients of the higher-order derivatives can be calculated from the following recurrence relationship [26]:

$$
A_{i k}^{(r)}=\left\{\begin{array}{c}
r\left[A_{i i}^{(r-1)} A_{i k}^{(1)}-\frac{A_{i k}^{(r-1)}}{X_{i}-X_{k}}\right] \\
i \neq k, \quad i, k=1,2, \cdots, n \\
-\sum_{\substack{j=1, j \neq i \\
i=k}}^{n} A_{i j}^{(r)} \quad i=1,2, \cdots, n
\end{array}\right.
$$

Let $Y_{1}, Y_{2}, \cdots, Y_{m}$ be a set of sampling points in the $Y$-direction. Using the quadrature rule, the sth-order $Y$-derivative of the vector $\{W(Y)\}$ at a sample point $Y=Y_{i}$ can be expressed as [51]:

$$
\begin{aligned}
& \frac{d^{s}}{d Y^{s}}\left\{\mathbf{W}\left(Y_{i}\right)\right\}=\sum_{j=1}^{m} B_{i k}^{(s)}\left\{\mathbf{W}\left(Y_{k}\right)\right\}, \\
& i=1,2, \cdots, m,
\end{aligned}
$$

where $B_{i k}^{(s)}$ is the sth-order $Y$-derivative weighting coefficient associated with the $Y=Y_{i}$ point, and:

$$
\left\{\mathbf{W}\left(Y_{i}\right)\right\}
$$

$$
\begin{aligned}
& =\left[\begin{array}{llll}
W\left(X_{1}, Y_{i}\right) & W\left(X_{2}, Y_{i}\right) & \cdots & W\left(X_{n}, Y_{i}\right)
\end{array}\right]^{T}, \\
& i=1,2, \cdots, m .
\end{aligned}
$$

$B_{i k}^{(1)}$ and $B_{i k}^{(s)}$ are given by:

$$
\begin{aligned}
& B_{i k}^{(1)}=\left\{\begin{array}{l}
\frac{\Pi\left(Y_{i}\right)}{\left(Y_{i}-Y_{k}\right) \Pi\left(Y_{k}\right)} \\
i \neq k, \quad i, k=1,2, \cdots, m \\
-\sum_{\substack{j=1, j \neq i \\
i=k,}}^{m} B_{i j}^{(1)} \\
i=1,2, \cdots, m
\end{array}\right.
\end{aligned}
$$

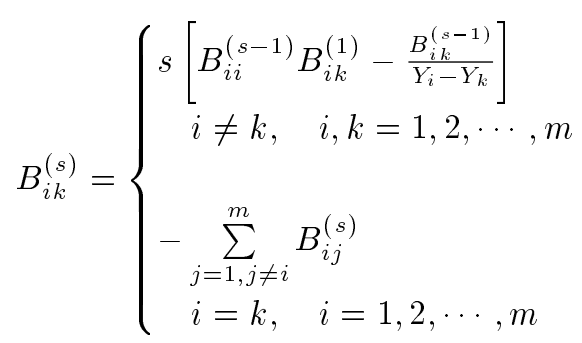

where:

$$
\Pi\left(Y_{i}\right)=\prod_{j=1, j \neq i}^{m}\left(Y_{i}-Y_{j}\right) .
$$

It is noted that the quadrature rule (Eq. (8)) can be written for all the $i$ values in the following matrix form:

$$
\frac{d^{r}}{d Y^{r}}\{\tilde{\mathbf{W}}\}=\left[\tilde{\mathbf{B}}^{(s)}\right]\{\tilde{\mathbf{W}}\},
$$

where:

$$
\begin{aligned}
& \{\tilde{\mathbf{w}}\} \\
& \left.=\left[\begin{array}{llll}
\left\{W\left(Y_{1}\right)\right\}^{T} & \left\{W\left(Y_{2}\right)\right\}^{T} & \cdots & \left\{W\left(Y_{m}\right)\right\}^{T}
\end{array}\right]^{T}\right], \\
& {\left[\tilde{\mathbf{B}}^{(s)}\right]=\left[\begin{array}{cccc}
B_{11}^{(s)}\left[\mathbf{I}^{x}\right] & B_{12}^{(s)}\left[\mathbf{I}^{x}\right] & \cdots & B_{1 m}^{(s)}\left[\mathbf{I}^{x}\right] \\
B_{21}^{(s)}\left[\mathbf{I}^{x}\right] & B_{22}^{(s)}\left[\mathbf{I}^{x}\right] & \cdots & B_{2 m}^{(s)}\left[\mathbf{I}^{x}\right] \\
\vdots & \vdots & \vdots & \vdots \\
B_{m 1}^{(s)}\left[\mathbf{I}^{x}\right] & B_{m 2}^{(s)}\left[\mathbf{I}^{x}\right] & \cdots & B_{m m}^{(s)}\left[\mathbf{I}^{x}\right]
\end{array}\right],}
\end{aligned}
$$

where $\left[\mathbf{I}^{x}\right]$ is an identity matrix of order $n \times n$.

\section{Governing equation and boundary conditions}

The governing differential equation for free vibration of an isotropic thin rectangular plate with length $a$ and width $b$ can be expressed as:

$$
W_{, X X X X}+2 \lambda^{2} W_{, X X Y Y}+\lambda^{4} W_{, Y Y Y Y}=\Omega^{2} W,
$$

where a subscript comma indicates partial differentiation; $W(X, Y)$ is the dimensionless mode function of the lateral deflection; $X=x / a$ and $Y=y / b$ are 
dimensionless coordinates; $\lambda=a / b$ is the aspect ratio; and $\Omega=\omega a^{2} \sqrt{\rho h / D}$ is the dimensionless frequency parameter, wherein $\omega, \rho, h$, and $D$ are, respectively, the circular frequency, mass density, thickness, and bending stiffness of the plate. The boundary conditions of the rectangular plate are:

(I) Simply-supported edge (S):

$$
\begin{aligned}
& W=W_{, X X}=0 \\
& \text { at } X=0 \text { and } / \text { or } \quad X=1, \\
& W=W_{, Y Y}=0 \\
& \text { at } Y=0 \text { and } / \text { or } Y=1 .
\end{aligned}
$$

(II) Clamped edge (C):

$$
\begin{aligned}
& W=W_{, X}=0 \\
& \text { at } X=0 \text { and } / \text { or } \quad X=1, \\
& W=W_{, Y}=0 \\
& \text { at } Y=0 \text { and } / \text { or } Y=1 .
\end{aligned}
$$

(III) Free edge (F):

$$
\begin{aligned}
W_{, X X X}+(2-\mu) \lambda^{2} W_{, X Y Y}=W_{, X X} \\
+\mu \lambda^{2} W_{, Y Y}=0, \\
\text { at } X=0 \quad \text { and } / \text { or } \quad X=1, \\
W_{, Y Y Y}+\frac{2-\mu}{\lambda^{2}} W_{, Y X X}=W_{, Y Y} \\
+\frac{\mu}{\lambda^{2}} W_{, X X}=0,
\end{aligned}
$$$$
\text { at } Y=0 \text { and/or } Y=1 \text {, }
$$

wherein $\mu$ is the Poisson's ratio. For a free corner formed by the intersection of two free edges, the following additional condition must also be satisfied at the corner [1]:

$$
W_{, X Y}=0 \text {. }
$$

\section{Proposed differential quadrature methodology}

The proposed methodology first reduces the original plate problem (governed by Eq. (16)) to two simple beam problems. Each beam problem is then solved using the DQM while the corresponding boundary conditions can be implemented separately. This significantly simplifies the solution procedure and its implementation as compared with the conventional procedure where the plate problem is directly solved using the DQM. The details of the proposed methodology are given in the following sub-sections.

\subsection{Procedure for the solution of the first beam problem}

4.1.1. DQM approximation of $X$-derivatives

Satisfying Eq. (16) at any sample point $X=X_{i}$, one has:

$$
\begin{aligned}
W_{, X X X X} & \left(X_{i}, Y\right)+2 \lambda^{2} W_{, X X Y Y}\left(X_{i}, Y\right) \\
& +\lambda^{4} W_{, Y Y Y Y}\left(X_{i}, Y\right)=\Omega^{2} W\left(X_{i}, Y\right), \\
i=1,2, \cdots, n &
\end{aligned}
$$

Substituting the quadrature rule, given in Eq. (1), with Eq. (24) gives:

$$
\begin{aligned}
& \sum_{j=1}^{n} A_{i j}^{(4)} W\left(X_{j}, Y\right)+2 \lambda^{2} \sum_{j=1}^{n} A_{i j}^{(2)} W_{, Y Y}\left(X_{j}, Y\right) \\
& \quad+\lambda^{4} W_{, Y Y Y Y}\left(X_{i}, Y\right)=\Omega^{2} W\left(X_{i}, Y\right), \\
& i=1,2, \cdots, n .
\end{aligned}
$$

Eq. (25) can also be expressed in matrix notation as:

$$
\begin{aligned}
{\left[\mathbf{A}^{(4)}\right] } & \{\mathbf{W}(Y)\}+2 \lambda^{2}\left[\mathbf{A}^{(2)}\right]\left\{\mathbf{W}_{, Y Y}(Y)\right\} \\
& +\lambda^{4}\left[\mathbf{I}^{x}\right]\left\{\mathbf{W}_{, Y Y Y Y}(Y)\right\}=\Omega^{2}\left[\mathbf{I}^{x}\right]\{\mathbf{W}(Y)\}
\end{aligned}
$$

where $\left[\mathbf{A}^{(4)}\right]$ and $\left[\mathbf{A}^{(2)}\right]$ are the fourth-order and second-order DQM weighting coefficient matrices, respectively; $\left[\mathbf{I}^{x}\right]$ is an identity matrix of order $n \times n$; and the vector $\{\mathbf{W}(Y)\}$ has already been defined in Eq. (3).

Eq. (26) represents a system of coupled ordinary differential equations of the fourth-order, which can be further discretized using the DQM. However, it is possible to impose the $X$-direction boundary conditions of the plate before applying the DQM to this system. The details are given in the next subsection.

\subsection{2. $D Q M$ analogs of the boundary conditions in the $X$-direction}

The boundary conditions of the rectangular plate in the $X$-direction are given in Eqs. (17), (19), and (21). The corresponding quadrature analogs are detailed below:

(I) Simply supported end condition at $X=X_{p}$ ( $p=$ 1 or $n$ ): From Eqs. (1) and (17), the quadrature analogs of the boundary conditions are obtained as follows:

$$
\begin{aligned}
& W\left(X_{p}, Y\right)=0, \\
& \sum_{j=1}^{n} A_{p j}^{(2)} W\left(X_{j}, Y\right)=0 .
\end{aligned}
$$


(II) Clamped end condition at $X=X_{p}(p=1$ or $n)$ : From Eqs. (1) and (19), the quadrature analogs of the boundary conditions are simply written as:

$$
\begin{aligned}
& W\left(X_{p}, Y\right)=0, \\
& \sum_{j=1}^{n} A_{p j}^{(1)} W\left(X_{j}, Y\right)=0 .
\end{aligned}
$$

(III) Free end condition at $X=X_{p}(p=1$ or $n)$ : From Eqs. (1) and (21), the quadrature analogs of the boundary conditions are written as:

$$
\begin{aligned}
& \sum_{j=1}^{n} A_{p j}^{(3)} W\left(X_{j}, Y\right) \\
& \quad+(2-\mu) \lambda^{2} \sum_{j=1}^{n} A_{p j}^{(1)} W_{, Y Y}\left(X_{j}, Y\right)=0 \\
& \sum_{j=1}^{n} A_{p j}^{(2)} W\left(X_{j}, Y\right)+\mu \lambda^{2} W_{, Y Y}\left(X_{p}, Y\right)=0 .
\end{aligned}
$$

\subsubsection{Implementation of boundary conditions in the $X$-direction}

At this step, the boundary conditions of the plate in the $X$-direction can be applied to Eq. (26). This can be done simply by direct substitution of the boundary analog equations corresponding to simply-supported, clamped, and free edges (given in Eqs. (27)-(30)) with Eq. (26). By doing so, we obtain the following system of ordinary differential equations:

$$
\begin{gathered}
{\left[\begin{array}{c}
{\left[\mathbf{A}^{(4)}\right]_{b l}} \\
{\left[\mathbf{A}^{(4)}\right]_{d}} \\
{\left[\mathbf{A}^{(4)}\right]_{b r}}
\end{array}\right]\{\mathbf{W}(Y)\}+2 \lambda^{2}\left[\begin{array}{l}
{\left[\mathbf{A}^{(2)}\right]_{b l}} \\
{\left[\mathbf{A}^{(2)}\right]_{d}} \\
{\left[\mathbf{A}^{(2)}\right]_{b r}}
\end{array}\right]\left\{\mathbf{W}_{, Y Y}(Y)\right\}} \\
+\lambda^{4}\left[\begin{array}{c}
{\left[\mathbf{I}^{x}\right]_{b l}} \\
{\left[\mathbf{I}^{x}\right]_{d}} \\
{\left[\mathbf{I}^{x}\right]_{b r}}
\end{array}\right]\left\{\mathbf{W}_{, Y Y Y Y}(Y)\right\} \\
=\Omega^{2}\left[\begin{array}{c}
{\left[\mathbf{I}^{x}\right]_{b l}} \\
{\left[\mathbf{I}^{x}\right]_{d}} \\
{\left[\mathbf{I}^{x}\right]_{b r}}
\end{array}\right]\{\mathbf{W}(Y)\}
\end{gathered}
$$

where the subscripts $b l, d$, and $b r$ denote left boundary points, domain points, and right boundary points, respectively. $\left[\mathbf{A}^{(4)}\right]_{b l},\left[\mathbf{A}^{(4)}\right]_{b r},\left[\mathbf{A}^{(2)}\right]_{b l}$, and $\left[\mathbf{A}^{(2)}\right]_{b r}$ are matrices depending on the boundary conditions of the plate in the $X$-direction (see Appendices A and B for details); $\left[\mathbf{I}^{x}\right]_{b l}$ and $\left[\mathbf{I}^{x}\right]_{b r}$ are zero matrices; and:

$$
\begin{aligned}
& {\left[\mathbf{A}^{(4)}\right]_{d}=\left[\begin{array}{cccc}
A_{31}^{(4)} & A_{32}^{(4)} & \cdots & A_{3 n}^{(4)} \\
A_{41}^{(4)} & A_{42}^{(4)} & \cdots & A_{4 n}^{(4)} \\
\vdots & \vdots & \vdots & \vdots \\
A_{(n-2) 1}^{(4)} & A_{(n-2) 2}^{(4)} & \cdots & A_{(n-2) n}^{(4)}
\end{array}\right] \text {, }} \\
& {\left[\mathbf{A}^{(2)}\right]_{d}=\left[\begin{array}{cccc}
A_{31}^{(2)} & A_{32}^{(2)} & \cdots & A_{3 n}^{(2)} \\
A_{41}^{(2)} & A_{42}^{(2)} & \cdots & A_{4 n}^{(2)} \\
\vdots & \vdots & \vdots & \vdots \\
A_{(n-2) 1}^{(2)} & A_{(n-2) 2}^{(2)} & \cdots & A_{(n-2) n}^{(2)}
\end{array}\right] \text {, }} \\
& {\left[\mathbf{I}^{x}\right]_{d}=\left[\begin{array}{cccc}
I_{31}^{x} & I_{32}^{x} & \cdots & I_{3 n}^{x} \\
I_{41}^{x} & I_{42}^{x} & \cdots & I_{4 n}^{x} \\
\vdots & \vdots & \vdots & \vdots \\
I_{(n-2) 1}^{x} & I_{(n-2) 2}^{x} & \cdots & I_{(n-2) n}^{x}
\end{array}\right]}
\end{aligned}
$$

After eliminating the degrees of freedom related to Dirichlet-type boundary conditions (if any exist), Eq. (31) takes the form:

$$
\begin{aligned}
& {\left[\hat{\mathbf{A}}^{(4)}\right]\{\hat{\mathbf{W}}(Y)\}+2 \lambda^{2}\left[\hat{\mathbf{A}}^{(2)}\right]\left\{\hat{\mathbf{W}}_{, Y Y}(Y)\right\}} \\
& \quad+\lambda^{4}\left[\hat{\mathbf{I}}^{x}\right]\left\{\hat{\mathbf{W}}_{, Y Y Y Y}(Y)\right\}=\Omega^{2}\left[\hat{\mathbf{I}}^{x}\right]\{\hat{\mathbf{W}}(Y)\} .
\end{aligned}
$$

Eq. (34) involves the quadrature analog equations of the plate boundary conditions in the $X$-direction. If we denote the order of this matrix equation by $n_{f}$, it can be easily verified that $n_{f}=n-n_{s}-n_{c}$, wherein $n_{s}$ is the number of simply supported edges in the $X$ direction and $n_{c}$ is the number of clamped edges in the $X$-direction. Therefore, in general, $n_{f} \leq n$, where $n$ is the size of matrix equation (26):

\subsection{Procedure for the solution of the second beam problem}

4.2.1. DQM approximation of $Y$-derivatives

Satisfying Eq. (34) at any sample point $Y=Y_{i}$, one has:

$$
\begin{aligned}
& {\left[\hat{\mathbf{A}}^{(4)}\right]\left\{\hat{\mathbf{W}}\left(Y_{i}\right)\right\}+2 \lambda^{2}\left[\hat{\mathbf{A}}^{(2)}\right]\left\{\hat{\mathbf{W}}_{, Y Y}\left(Y_{i}\right)\right\}} \\
& \quad+\lambda^{4}\left[\hat{\mathbf{I}}^{x}\right]\left\{\hat{\mathbf{W}}_{, Y Y Y Y}\left(Y_{i}\right)\right\}=\Omega^{2}\left[\hat{\mathbf{I}}^{x}\right]\left\{\hat{\mathbf{W}}\left(Y_{i}\right)\right\} \\
& i=1,2, \cdots, m .
\end{aligned}
$$


Substituting the quadrature rule, given in Eq. (8), with Eq. (35) gives:

$$
\begin{aligned}
& {\left[\hat{\mathbf{A}}^{(4)}\right]\left\{\hat{\mathbf{W}}\left(Y_{i}\right)\right\}+2 \lambda^{2}\left[\hat{\mathbf{A}}^{(2)}\right] \sum_{j=1}^{m} B_{i j}^{(2)}\left\{\hat{\mathbf{W}}\left(Y_{j}\right)\right\}} \\
& +\lambda^{4}\left[\hat{\mathbf{I}}^{x}\right] \sum_{j=1}^{m} B_{i j}^{(4)}\left\{\hat{\mathbf{W}}\left(Y_{j}\right)\right\}=\Omega^{2}\left[\hat{\mathbf{I}}^{x}\right]\left\{\hat{\mathbf{W}}\left(Y_{i}\right)\right\}, \\
& i=1,2, \cdots, m .
\end{aligned}
$$

Eq. (36) can be written for all the $i$-values in the compact form:

$$
[\tilde{\mathbf{K}}]\{\tilde{\mathbf{W}}\}=\Omega^{2}[\tilde{\mathbf{M}}]\{\tilde{\mathbf{W}}\}
$$

where $[\tilde{\mathbf{K}}]$ is the stiffness matrix, $[\tilde{\mathbf{M}}]$ is the mass matrix, and $\{\tilde{\mathbf{W}}\}$ is the unknown coefficient vector. The $n_{f} \times n_{f}$ sub-matrices $\left[\tilde{\mathbf{K}}_{i j}\right]$ and $\left[\tilde{\mathbf{M}}_{i j}\right]$ are 2 given by:

$$
\begin{aligned}
{\left[\tilde{\mathbf{K}}_{i j}\right] } & =I_{i j}^{y}\left[\hat{\mathbf{A}}^{4}\right]+2 \lambda^{2} B_{i j}^{(2)}\left[\hat{\mathbf{A}}^{(2)}\right]+\lambda^{4} B_{i j}^{(4)}\left[\hat{I}^{x}\right], \\
i, j & =1,2, \cdots, m \\
{\left[\tilde{\mathbf{M}}_{i j}\right] } & =I_{i j}^{y}\left[\hat{\mathbf{I}}^{x}\right]
\end{aligned}
$$

where $I_{i j}^{y}$ is the element of $m \times m$ identity matrix, and:

$$
\begin{aligned}
& \{\tilde{\mathbf{W}}\} \\
& \quad=\left[\left\{\hat{\mathbf{W}}\left(Y_{1}\right)\right\}^{T}\left\{\hat{\mathbf{W}}\left(Y_{2}\right)\right\}^{T} \cdots\left\{\hat{\mathbf{W}}\left(Y_{m}\right)\right\}^{T}\right]_{(40)}^{T} .
\end{aligned}
$$

It is noted that the size of mass and stiffness matrices in Eq. (37) is $m n_{f} \times m n_{f}$, where $n_{f}=n-n_{s}-n_{c}$. The eigenvalue problem (Eq. 37) can be solved for the eigenvalues $\Omega$, if the boundary conditions of the plate problem in $Y$-direction are also applied. The procedure will be detailed in the next section.

\subsubsection{DQM analogs of the boundary conditions in the $Y$-direction}

The boundary conditions of the rectangular plate in $Y$-direction are given in Eqs. (18), (20), and (22). The corresponding quadrature analogs are detailed below:

(I) Simply supported end condition at $Y=Y_{q}(q=1$ or $m$ ): From Eqs. (8) and (18), the quadrature analogs of the boundary conditions are obtained as follows:

$$
\begin{aligned}
& \left\{\hat{\mathbf{W}}\left(Y_{q}\right)\right\}=\{\mathbf{0}\}_{n_{f} \times 1}, \\
& \left\{\hat{\mathbf{W}}_{, Y Y}\left(Y_{q}\right)\right\}=\sum_{j=1}^{m} B_{q j}^{(2)}\left\{\hat{\mathbf{W}}\left(Y_{j}\right)\right\}=\{\mathbf{0}\}_{n_{f} \times 1} .
\end{aligned}
$$

(II) Clamped end condition at $Y=Y_{q}(q=1$ or $m)$ : From Eqs. (8) and (20), the quadrature analogs of the boundary conditions are simply written as:

$$
\begin{aligned}
& \left\{\hat{\mathbf{W}}\left(Y_{q}\right)\right\}=\{0\}_{n_{f} \times 1} \\
& \left\{\hat{\mathbf{W}}_{, Y}\left(Y_{q}\right)\right\}=\sum_{j=1}^{m} B_{q j}^{(1)}\left\{\hat{\mathbf{W}}\left(Y_{j}\right)\right\}=\{\mathbf{0}\}_{n_{f} \times 1}
\end{aligned}
$$

(III) Free end condition at $Y=Y_{q}(q=1$ or $m)$ : Substituting the quadrature rule (given in Eq. (2)) with Eq. (22) gives:

$$
\begin{aligned}
& \left\{\mathbf{W}_{, Y Y Y}\left(Y_{q}\right)\right\}+\frac{2-\mu}{\lambda^{2}}\left[\mathbf{A}^{(2)}\right]\left\{\mathbf{W}_{, Y}\left(Y_{q}\right)\right\} \\
& =\{\mathbf{0}\}_{n \times 1}, \\
& \left\{\mathbf{W}_{, Y Y}\left(Y_{q}\right)\right\}+\frac{\mu}{\lambda^{2}}\left[\mathbf{A}^{(2)}\right]\left\{\mathbf{W}\left(Y_{q}\right)\right\}=\{\mathbf{0}\}_{n \times 1} .
\end{aligned}
$$

By eliminating the degrees of freedom related to Dirichlet-type boundary conditions (if any exist), Eqs. (43) and (44) may be rewritten as:

$$
\begin{aligned}
\left\{\hat{\mathbf{W}}_{, Y Y Y}\left(Y_{q}\right)\right\} & +\frac{2-\mu}{\lambda^{2}}\left[\tilde{\mathbf{A}}^{(2)}\right]\left\{\hat{\mathbf{W}}_{, Y}\left(Y_{q}\right)\right\} \\
& =\{\boldsymbol{0}\}_{n_{f} \times 1} \\
\left\{\hat{\mathbf{W}}_{, Y Y}\left(Y_{q}\right)\right\} & +\frac{\mu}{\lambda^{2}}\left[\tilde{\mathbf{A}}^{(2)}\right]\left\{\hat{\mathbf{W}}\left(Y_{q}\right)\right\} \\
& =\{\boldsymbol{0}\}_{n_{f} \times 1} .
\end{aligned}
$$

Now, using the quadrature rule given in Eq. (8), Eqs. (45) and (46) may be written as:

$$
\begin{aligned}
& \sum_{j=1}^{m} B_{q j}^{(3)}\left\{\hat{\mathbf{W}}\left(Y_{j}\right)\right\} \\
& \quad+\frac{2-\mu}{\lambda^{2}}\left[\tilde{\mathbf{A}}^{(2)}\right] \sum_{j=1}^{m} B_{q j}^{(1)}\left\{\hat{\mathbf{W}}\left(Y_{j}\right)\right\} \\
& \quad=\{\mathbf{0}\}_{n_{f} \times 1}, \\
& \sum_{j=1}^{m} B_{q j}^{(2)}\left\{\hat{\mathbf{W}}\left(Y_{j}\right)\right\}+\frac{\mu}{\lambda^{2}}\left[\tilde{\mathbf{A}}^{(2)}\right]\left\{\hat{\mathbf{W}}\left(Y_{q}\right)\right\} \\
& =\{\mathbf{0}\}_{n_{f} \times 1} .
\end{aligned}
$$

(IV) Free corner boundary condition at $\left(X_{p}, Y_{q}\right)$ : As pointed out earlier, the condition $W_{, X Y}=0$ must 
also be applied at free corners. First, we note that:

$$
\begin{aligned}
& \frac{\partial W\left(X_{p}, Y\right)}{\partial X}=\sum_{j=1}^{n} A_{p j}^{(1)} W\left(X_{j}, Y\right) \\
& \quad=\left[\begin{array}{llll}
A_{p 1}^{(1)} & A_{p 2}^{(2)} & \cdots & A_{p n}^{(1)}
\end{array}\right]\left\{\begin{array}{c}
W\left(X_{1}, Y\right) \\
W\left(X_{2}, Y\right) \\
\vdots \\
W\left(X_{n}, Y\right)
\end{array}\right\} \\
& =\left[\mathbf{A}_{p}\right]_{1 \times n}\{\mathbf{W}(Y)\}_{n \times 1},
\end{aligned}
$$

also:

$$
\begin{aligned}
\frac{\partial^{2} W\left(X_{p}, Y_{q}\right)}{\partial X \partial Y} & =\frac{\partial}{\partial Y}\left(\frac{\partial W\left(X_{p}, Y\right)}{\partial X}\right)_{Y=Y_{q}} \\
& =\left[\mathbf{A}_{p}\right]\left\{\mathbf{W}_{, Y}\left(Y_{q}\right)\right\} .
\end{aligned}
$$

Now, using the quadrature rule given in Eq. (8), the quadrature analog of the corner boundary condition is obtained as:

$$
\frac{\partial^{2} W\left(X_{p}, Y_{q}\right)}{\partial X \partial Y}=\left[\mathbf{A}_{p}\right] \sum_{j=1}^{m} B_{q j}^{(1)}\left\{\mathbf{W}\left(Y_{j}\right)\right\}=0 .
$$

If the degrees of freedom related to Dirichlet-type boundary conditions (in the $X$-direction) are also eliminated, Eq. (51) can be expressed as:

$$
\frac{\partial^{2} W\left(X_{p}, Y_{q}\right)}{\partial X \partial Y}=\left[\hat{\mathbf{A}}_{p}\right] \sum_{j=1}^{m} B_{q j}^{(1)}\left\{\hat{\mathbf{W}}\left(Y_{j}\right)\right\}=0
$$

\subsubsection{Implementation of boundary conditions in the $Y$-direction}

The procedure for implementing the boundary conditions of the plate problem in $Y$-direction is similar to that presented in Section 4.1.3. But, the procedure for plates with free corners differs slightly from that of plates without free corners, as we will show in the following subsections.

\subsubsection{Procedure for plates without free corners}

Substituting the boundary analog equations corresponding to simply-supported, clamped, and free edges (given in Section 4.2.2) with Eq. (37) leads to the following eigenvalue equation:

$$
\left[\begin{array}{c}
{\left[\tilde{\mathbf{K}}_{b l}\right]} \\
{\left[\tilde{\mathbf{K}}_{d}\right]} \\
{\left[\tilde{\mathbf{K}}_{b r}\right]}
\end{array}\right]\{\tilde{\mathbf{W}}\}=\Omega^{2}\left[\begin{array}{c}
{\left[\tilde{\mathbf{M}}_{b l}\right]} \\
{\left[\tilde{\mathbf{M}}_{d}\right]} \\
{\left[\tilde{\mathbf{M}}_{b r}\right]}
\end{array}\right]\{\tilde{\mathbf{W}\}},
$$

where the subscripts $b l, d$, and $b r$ denote left boundary points, domain points, and right boundary points, respectively. $\left[\tilde{\mathbf{K}}_{b l}\right]$ and $\left[\tilde{\mathbf{K}}_{b r}\right]$ are matrices depending on boundary conditions of the plate in $Y$-direction (see Appendices $\mathrm{C}$ and $\mathrm{D}$ for details); $\left[\tilde{\mathbf{M}}_{b l}\right]$ and $\left[\tilde{\mathbf{M}}_{b r}\right]$ are zero matrices, and:

$$
\begin{aligned}
& {\left[\tilde{\mathbf{K}}_{d}\right]=\left[\begin{array}{cccc}
{\left[\tilde{\mathbf{K}}_{31}\right]} & {\left[\tilde{\mathbf{K}}_{32}\right]} & \cdots & {\left[\tilde{\mathbf{K}}_{3 m}\right]} \\
{\left[\tilde{\mathbf{K}}_{41}\right]} & {\left[\tilde{\mathbf{K}}_{42}\right]} & \cdots & {\left[\tilde{\mathbf{K}}_{4 m}\right]} \\
\vdots & \vdots & \vdots & \vdots \\
{\left[\tilde{\mathbf{K}}_{(m-2) 1}\right]} & {\left[\tilde{\mathbf{K}}_{(m-2) 2}\right]} & \cdots & {\left[\tilde{\mathbf{K}}_{(m-2) m}\right]}
\end{array}\right],} \\
& {\left[\tilde{\mathbf{M}}_{d}\right]=\left[\begin{array}{cccc}
{\left[\tilde{\mathbf{M}}_{31}\right]} & {\left[\tilde{\mathbf{M}}_{32}\right]} & \cdots & {\left[\tilde{\mathbf{M}}_{3 m}\right]} \\
{\left[\tilde{\mathbf{M}}_{41}\right]} & {\left[\tilde{\mathbf{M}}_{42}\right]} & \cdots & {\left[\tilde{\mathbf{M}}_{4 m}\right]} \\
\vdots & \vdots & \vdots & \vdots \\
{\left[\tilde{\mathbf{M}}_{(m-2) 1}\right]\left[\tilde{\mathbf{M}}_{(m-2) 2}\right]} & \cdots & {\left[\tilde{\mathbf{M}}_{(m-2) m}\right]}
\end{array}\right]}
\end{aligned}
$$

For some cases, Eq. (53) can be directly solved for the eigenvalues. However, in general, the eigenvalue problem (Eq. (53)) is highly ill-conditioned and cannot be easily solved for the eigenvalues. A way for overcoming this issue is to eliminate the degrees of freedom corresponding to the Dirichlet-type boundary conditions. By doing so, the ill-conditioned eigenvalue problem (Eq. (53)) is converted to a well-conditioned eigenvalue problem. It is noted that the size of resulting eigenvalue equations is $m_{f} n_{f} \times m_{f} n_{f}$, where $m_{f}=$ $m-m_{s}-m_{c}$ and $n_{f}=n-n_{s}-n_{c}$; wherein $n_{s}$ is the number of simply supported edges in the $X$-direction, $n_{c}$ is the number of clamped edges in the $X$-direction, $m_{s}$ is the number of simply supported edges in the $Y$ direction, and $m_{c}$ is the number of clamped edges in the $Y$-direction. It is also noted that the resultant mass matrix of the eigenvalue problem (Eq. (53)) involves some zero rows and hence is singular. But, such eigenvalue problem can be easily solved using the QZ algorithm [52], of which the programs and subroutines are available in most linear algebra software packages such as MATLAB and LAPACK.

\subsubsection{Procedure for plates with free corners}

The solution procedure for plates involving free corners is similar to that presented in Section 4.2.3.1. But, it involves an additional step. In this case, as it was pointed out earlier, the additional boundary analog equation (52) must also be imposed on the system of discrete equations (53). 
In the very first glance, it may appear that the free corner boundary analog equation is arbitrarily substituted in the system of discrete (Eqs. (53)). In this regard, there are different choices for replacement of the quadrature analog equations of the governing differential equation by the quadrature analog equation of the free corner boundary condition. Noting that the plate has a free corner at $\left(X_{p}, Y_{q}\right)$, a natural way is to impose the free corner boundary condition at this point (free corner point). In other words, the quadrature analog equation of the governing differential equation at $\left(X_{p}, Y_{q}\right)$ can be replaced by the quadrature analog equation of the free corner boundary condition.

\section{Numerical results}

To demonstrate the stability, rate of convergence, and accuracy of the proposed DQM methodology, natural frequencies of rectangular plates with different boundary conditions are evaluated and the results are tabulated in Tables 1-5. To simplify the notation, the edge conditions for plates are denoted by letters S (simply supported), C (clamped), and F (free). For instance, SCSF denotes that the plate has a simply supported edge at $X=0$, a clamped edge at $Y=0$, a simply supported edge at $X=1$, and a free edge at $Y=1$.

For the DQM solution of the present problem, we considered a grid of $n \times m$ sampling points obtained by taking $n$ and $m$ points in $0 \leq X \leq 1$ and $0 \leq Y \leq$ 1 , respectively. Moreover, the DQM sampling points are taken non-uniformly spaced and are given by the following equations:

$$
\begin{aligned}
& X_{1}=0, \quad X_{2}=\delta, \quad X_{n-1}=1-\delta, \quad X_{n}=1 \\
& X_{i}=\frac{1}{2}\left[1-\cos \left(\frac{(i-2) \pi}{n-3}\right)\right] \\
& i=3,4, \cdots, n-2
\end{aligned}
$$

and similar equations for the $Y$-direction sampling points. Here, $X_{2}$ and $X_{n-1}$ are discrete points very close to the boundary points (adjacent $\delta$-points). The parameter $\delta$ shows the closeness of the adjacent point and the respective boundary point. In order to achieve accurate solutions by using this type of sampling points, the magnitude of $\delta$ should be as small as possible $\left(\leq 10^{-3}\right)$. In this study, the magnitude of parameter $\delta$ is assumed to be $\delta=10^{-3}$.

Table 1 shows the convergence study of the first five dimensionless natural frequencies of Levy-type square plates (i.e., plates with two opposite sides simply supported). The number of sampling points in the $X$ and $Y$ directions (i.e., $n$ and $m$ ) are taken to be the same (i.e., we assumed that $n=m$ ). The analytical solutions of Leissa [1] are also shown in this table for comparison purposes. It can be clearly seen from Table 1 that the present results converge very quickly and agree very closely with the exact solution values of Leissa [1], even with all the available significant digits.

The first five non-dimensional frequency parameters for square plates involving free corners are tabulated in Tables 2 and 3. These results are obtained by considering an equal number of sampling points in both $X$ and $Y$ directions $(n=m)$. The results are also compared with the results obtained by the conventional Ritz method [1], new Ritz formulation [5], Generalized Differential Quadrature e Method (GDQM) [22], and the FE-Ritz method [53]. It is noted that the results of Eftekhari and Jafari in $[5,53]$ are believed to be highly accurate since both the geometric and natural boundary conditions of the plate are strongly satisfied in the algorithms presented in these references. Comparing the results of Table 2 with those of Table 1 , it can be seen that the rate of convergence of the proposed DQM methodology for plates with free corners is not as high as those for plates without free corners. It can also be seen from Tables 2 and 3 that, in most cases, the present solutions converge to values less than those given in $[1,5,22,53]$. Noting that the results given in $[1,5,53]$ are upper bounds of the analytical values, it can be concluded that the present solutions are often closer to the exact values of the natural frequencies than those in $[1,5,53]$. The results of Shu and Du [22] are found to be somewhat oscillatory. For instance, while some results of them [22] are very close to those of Eftekhari and Jafari in [5,53], some others do not show the same trend and do not agree well with the results of these references. This oscillatory behavior is due to the lack of satisfaction of the free corner boundary conditions in the GQDM formulation of Shu and Du [22].

To better see the convergence behavior and accuracy of the proposed DQM, the variation of the percent error in quadrature solutions (defined as $\mid \Omega_{\mathrm{DQM}}-$ $\left.\Omega_{\text {Ritz [5] }} / \Omega_{\text {Ritz [5] }} \times 100\right)$ with respect to the number of sampling points is shown in Figure 1. It can be seen from Figure 1 that the solutions obtained by the present DQM, in most cases, show a monotonic convergence with increasing number of sampling points. However, the speed of convergence is very slow in some cases, particularly in the results for the fundamental frequencies of plates with CCFF, CSFF, and CFFF boundary conditions. Therefore, the proposed DQM requires a large computational cost to obtain sufficient accuracy for these cases. In solving the free vibration problem of plates with irregular geometries, Bert and Malik [54] have also reported this convergence problem and have found that this 
Table 1. Convergence and comparison of natural frequencies of Levy-type square plates.

\begin{tabular}{|c|c|c|c|c|c|c|}
\hline Plate & $n=m$ & $\Omega_{1}$ & $\Omega_{2}$ & $\Omega_{3}$ & $\Omega_{4}$ & $\Omega_{5}$ \\
\hline \multirow{6}{*}{ SSSS } & 11 & 19.7392 & 49.3489 & 49.3489 & 78.9580 & 98.6268 \\
\hline & 13 & 19.7392 & 49.3480 & 49.3480 & 78.9568 & 98.6948 \\
\hline & 15 & 19.7392 & 49.3480 & 49.3480 & 78.9568 & 98.6961 \\
\hline & 17 & 19.7392 & 49.3480 & 49.3480 & 78.9568 & 98.6960 \\
\hline & 19 & 19.7392 & 49.3480 & 49.3480 & 78.9568 & 98.6960 \\
\hline & Exact [1] & 19.7392 & 49.3480 & 49.3480 & 78.9568 & 98.6960 \\
\hline \multirow{6}{*}{ SCSS } & 11 & 23.6463 & 51.6753 & 58.6481 & 86.1364 & 100.2023 \\
\hline & 13 & 23.6463 & 51.6743 & 58.6463 & 86.1345 & 100.2686 \\
\hline & 15 & 23.6463 & 51.6743 & 58.6464 & 86.1345 & 100.2698 \\
\hline & 17 & 23.6463 & 51.6743 & 58.6464 & 86.1345 & 100.2698 \\
\hline & 19 & 23.6463 & 51.6743 & 58.6464 & 86.1345 & 100.2698 \\
\hline & Exact [1] & 23.6463 & 51.6743 & 58.6464 & 86.1345 & 100.2698 \\
\hline \multirow{6}{*}{ SCSC } & 11 & 28.9509 & 54.7441 & 69.3317 & 94.5904 & 102.1501 \\
\hline & 13 & 28.9509 & 54.7431 & 69.3271 & 94.5854 & 102.2150 \\
\hline & 15 & 28.9509 & 54.7431 & 69.3270 & 94.5853 & 102.2162 \\
\hline & 17 & 28.9509 & 54.7431 & 69.3270 & 94.5853 & 102.2162 \\
\hline & 19 & 28.9509 & 54.7431 & 69.3270 & 94.5853 & 102.2162 \\
\hline & Exact [1] & 28.9509 & 54.7431 & 69.3270 & 94.5853 & 102.2162 \\
\hline \multirow{6}{*}{ SSSF } & 11 & 11.6846 & 27.7570 & 41.2023 & 59.0790 & 61.8910 \\
\hline & 13 & 11.6845 & 27.7564 & 41.1969 & 59.0662 & 61.8600 \\
\hline & 15 & 11.6845 & 27.7563 & 41.1967 & 59.0655 & 61.8606 \\
\hline & 17 & 11.6845 & 27.7563 & 41.1967 & 59.0655 & 61.8606 \\
\hline & 19 & 11.6845 & 27.7563 & 41.1967 & 59.0655 & 61.8606 \\
\hline & Exact [1] & 11.6845 & 27.7563 & 41.1967 & 59.0655 & 61.8606 \\
\hline \multirow{6}{*}{ SCSF } & 11 & 12.6874 & 33.0666 & 41.7068 & 63.0375 & 72.4757 \\
\hline & 13 & 12.6874 & 33.0652 & 41.7021 & 63.0161 & 72.3948 \\
\hline & 15 & 12.6874 & 33.0651 & 41.7019 & 63.0149 & 72.3977 \\
\hline & 17 & 12.6874 & 33.0651 & 41.7019 & 63.0148 & 72.3976 \\
\hline & 19 & 12.6874 & 33.0651 & 41.7019 & 63.0148 & 72.3976 \\
\hline & Exact [1] & 12.6874 & 33.0651 & 41.7019 & 63.0148 & 72.3976 \\
\hline \multirow{6}{*}{ SFSF } & 11 & 9.6314 & 16.1353 & 36.7264 & 38.9483 & 46.7564 \\
\hline & 13 & 9.6314 & 16.1348 & 36.7257 & 38.9450 & 46.7390 \\
\hline & 15 & 9.6314 & 16.1348 & 36.7256 & 38.9450 & 46.7382 \\
\hline & 17 & 9.6314 & 16.1348 & 36.7256 & 38.9450 & 46.7381 \\
\hline & 19 & 9.6314 & 16.1348 & 36.7256 & 38.9450 & 46.7381 \\
\hline & Exact [1] & 9.6314 & 16.1348 & 36.7256 & 38.9450 & 46.7381 \\
\hline
\end{tabular}


Table 2. Convergence and comparison of natural frequencies of square plates involving free corners.

\begin{tabular}{|c|c|c|c|c|c|c|}
\hline Plate & $n=m$ & $\Omega_{1}$ & $\Omega_{2}$ & $\Omega_{3}$ & $\Omega_{4}$ & $\Omega_{5}$ \\
\hline \multirow{10}{*}{ SSFF } & 11 & 3.365 & 17.317 & 19.291 & 38.216 & 51.074 \\
\hline & 13 & 3.365 & 17.316 & 19.289 & 38.208 & 51.035 \\
\hline & 15 & 3.365 & 17.316 & 19.289 & 38.208 & 51.035 \\
\hline & 17 & 3.366 & 17.316 & 19.290 & 38.208 & 51.035 \\
\hline & 19 & 3.366 & 17.316 & 19.290 & 38.208 & 51.035 \\
\hline & 21 & 3.366 & 17.316 & 19.290 & 38.208 & 51.035 \\
\hline & New Ritz [5] & 3.3670 & 17.316 & 19.293 & 38.211 & 51.035 \\
\hline & FE-Ritz [53] & 3.3670 & 17.3164 & 19.2929 & 38.2112 & 51.0354 \\
\hline & Conventional Ritz [1] & 3.3687 & 17.407 & 19.367 & 38.291 & 51.324 \\
\hline & $\mathrm{GDQM}^{\mathrm{a}}[22]$ & 3.363 & 17.317 & 19.293 & 38.218 & 51.032 \\
\hline \multirow{16}{*}{ CSFF } & 11 & 5.522 & 19.201 & 24.464 & 42.954 & 52.748 \\
\hline & 13 & 5.463 & 19.168 & 24.524 & 42.964 & 52.714 \\
\hline & 15 & 5.428 & 19.146 & 24.564 & 42.984 & 52.716 \\
\hline & 17 & 5.405 & 19.130 & 24.592 & 43.003 & 52.716 \\
\hline & 19 & 5.390 & 19.118 & 24.612 & 43.019 & 52.715 \\
\hline & 21 & 5.379 & 19.109 & 24.626 & 43.032 & 52.714 \\
\hline & 23 & 5.371 & 19.102 & 24.637 & 43.043 & 52.714 \\
\hline & 25 & 5.365 & 19.096 & 24.645 & 43.051 & 52.713 \\
\hline & 27 & 5.361 & 19.092 & 24.651 & 43.058 & 52.712 \\
\hline & 29 & 5.358 & 19.088 & 24.655 & 43.063 & 52.711 \\
\hline & 31 & 5.355 & 19.085 & 24.659 & 43.067 & 52.711 \\
\hline & 33 & 5.354 & 19.083 & 24.662 & 43.071 & 52.710 \\
\hline & New Ritz [5] & 5.351 & 19.075 & 24.671 & 43.088 & 52.707 \\
\hline & FE-Ritz [53] & 5.3511 & 19.0752 & 24.6705 & 43.0876 & 52.7075 \\
\hline & Conventional Ritz [1] & 5.364 & 19.171 & 24.768 & 43.191 & 53.000 \\
\hline & $\mathrm{GDQM}^{\mathrm{a}}[22]$ & 5.402 & 19.219 & 25.005 & 43.372 & 52.702 \\
\hline \multirow{16}{*}{$\mathrm{CCFF}$} & 11 & 7.102 & 23.891 & 26.486 & 47.574 & 62.845 \\
\hline & 13 & 7.048 & 23.883 & 26.514 & 47.547 & 62.727 \\
\hline & 15 & 7.017 & 23.885 & 26.531 & 47.551 & 62.719 \\
\hline & 17 & 6.997 & 23.888 & 26.544 & 47.562 & 62.714 \\
\hline & 19 & 6.982 & 23.892 & 26.552 & 47.573 & 62.711 \\
\hline & 21 & 6.971 & 23.894 & 26.559 & 47.584 & 62.709 \\
\hline & 23 & 6.962 & 23.896 & 26.563 & 47.592 & 62.708 \\
\hline & 25 & 6.955 & 23.897 & 26.567 & 47.600 & 62.707 \\
\hline & 27 & 6.950 & 23.898 & 26.570 & 47.606 & 62.707 \\
\hline & 29 & 6.946 & 23.899 & 26.572 & 47.611 & 62.706 \\
\hline & 31 & 6.942 & 23.900 & 26.574 & 47.615 & 62.706 \\
\hline & 33 & 6.939 & 23.900 & 26.575 & 47.619 & 62.706 \\
\hline & New Ritz [5] & 6.919 & 23.904 & 26.585 & 47.651 & 62.706 \\
\hline & FE-Ritz [53] & 6.9195 & 23.9040 & 26.5851 & 47.6519 & 62.7063 \\
\hline & Conventional Ritz [1] & 6.942 & 24.034 & 26.681 & 47.785 & 63.039 \\
\hline & $\mathrm{GDQM}^{\mathrm{a}}[22]$ & 6.982 & 24.193 & 26.683 & 47.909 & 62.489 \\
\hline
\end{tabular}

a: Solutions with stretched sampling points. 
Table 3. Convergence and comparison of natural frequencies of square plates involving free corners.

\begin{tabular}{|c|c|c|c|c|c|c|}
\hline Plate & $\boldsymbol{n}=\boldsymbol{m}$ & $\Omega_{1}$ & $\Omega_{2}$ & $\Omega_{3}$ & $\Omega_{4}$ & $\Omega_{5}$ \\
\hline \multirow{11}{*}{ SFFF } & 11 & 6.638 & 14.902 & 25.378 & 25.997 & 48.466 \\
\hline & 13 & 6.639 & 14.901 & 25.371 & 25.995 & 48.445 \\
\hline & 15 & 6.640 & 14.901 & 25.370 & 25.996 & 48.447 \\
\hline & 17 & 6.641 & 14.901 & 25.371 & 25.996 & 48.448 \\
\hline & 19 & 6.641 & 14.901 & 25.371 & 25.997 & 48.448 \\
\hline & 21 & 6.642 & 14.901 & 25.372 & 25.998 & 48.449 \\
\hline & 23 & 6.642 & 14.901 & 25.372 & 25.998 & 48.449 \\
\hline & New Ritz [5] & 6.6437 & 14.9015 & 25.3757 & 26.0005 & 48.4495 \\
\hline & FE-Ritz [53] & 6.6437 & 14.9015 & 25.3757 & 26.0005 & 48.4495 \\
\hline & Conventional Ritz [1] & 6.6480 & 15.023 & 25.492 & 26.126 & 48.711 \\
\hline & $\mathrm{GDQM}^{\mathrm{a}}[22]$ & 6.636 & 14.901 & 25.388 & 26.003 & 48.469 \\
\hline \multirow{16}{*}{ CFFF } & 11 & 3.606 & 8.780 & 21.121 & 27.334 & 30.666 \\
\hline & 13 & 3.545 & 8.710 & 21.174 & 27.307 & 30.715 \\
\hline & 15 & 3.513 & 8.660 & 21.207 & 27.287 & 30.764 \\
\hline & 17 & 3.495 & 8.624 & 21.228 & 27.271 & 30.805 \\
\hline & 19 & 3.484 & 8.597 & 21.243 & 27.258 & 30.838 \\
\hline & 21 & 3.477 & 8.576 & 21.254 & 27.247 & 30.863 \\
\hline & 23 & 3.472 & 8.560 & 21.261 & 27.238 & 30.882 \\
\hline & 25 & 3.470 & 8.548 & 21.267 & 27.231 & 30.897 \\
\hline & 27 & 3.468 & 8.539 & 21.271 & 27.225 & 30.909 \\
\hline & 29 & 3.467 & 8.531 & 21.274 & 27.221 & 30.918 \\
\hline & 31 & 3.466 & 8.525 & 21.276 & 27.217 & 30.926 \\
\hline & 33 & 3.466 & 8.521 & 21.278 & 27.214 & 30.932 \\
\hline & New Ritz [5] & 3.4712 & 8.5074 & 21.2864 & 27.1990 & 30.9590 \\
\hline & FE-Ritz [53] & 3.4711 & 8.5067 & 21.2850 & 27.1989 & 30.9563 \\
\hline & Conventional Ritz [1] & 3.9417 & 8.5246 & 21.429 & 27.331 & 31.111 \\
\hline & $\mathrm{GDQM}^{\mathrm{a}}[22]$ & 3.485 & 8.604 & 21.586 & 27.230 & 31.358 \\
\hline \multirow{16}{*}{ FFFF } & 11 & 13.458 & 19.595 & 24.269 & 34.802 & 34.817 \\
\hline & 13 & 13.457 & 19.596 & 24.267 & 34.788 & 34.797 \\
\hline & 15 & 13.458 & 19.596 & 24.267 & 34.788 & 34.794 \\
\hline & 17 & 13.460 & 19.596 & 24.267 & 34.789 & 34.794 \\
\hline & 19 & 13.461 & 19.596 & 24.268 & 34.791 & 34.794 \\
\hline & 21 & 13.462 & 19.596 & 24.268 & 34.792 & 34.795 \\
\hline & 23 & 13.463 & 19.596 & 24.268 & 34.793 & 34.795 \\
\hline & 25 & 13.463 & 19.596 & 24.268 & 34.794 & 34.796 \\
\hline & 27 & 13.464 & 19.596 & 24.269 & 34.795 & 34.796 \\
\hline & 29 & 13.464 & 19.596 & 24.269 & 34.796 & 34.797 \\
\hline & 31 & 13.465 & 19.596 & 24.269 & 34.796 & 34.797 \\
\hline & 33 & 13.465 & 19.596 & 24.269 & 34.797 & 34.797 \\
\hline & New Ritz [5] & 13.4682 & 19.5961 & 24.2702 & 34.8009 & 34.8009 \\
\hline & FE-Ritz [53] & 13.4682 & 19.5961 & 24.2702 & 34.8009 & 34.8009 \\
\hline & Conventional Ritz [1] & 13.489 & 19.789 & 24.432 & 35.024 & 35.024 \\
\hline & $\mathrm{GDQM}^{\mathrm{a}}[22]$ & 13.454 & 19.597 & 24.271 & 34.815 & 34.817 \\
\hline
\end{tabular}

a: Solutions with stretched sampling points. 
Table 4. Convergence and comparison of natural frequencies of square plates with free corners (when non-uniform sampling points without adjacent $\delta$-points are used in the algorithm).

\begin{tabular}{|c|c|c|c|c|c|c|c|}
\hline Plate & Method & $n=m$ & $\Omega_{1}$ & $\Omega_{2}$ & $\Omega_{3}$ & $\Omega_{4}$ & $\Omega_{5}$ \\
\hline \multirow{8}{*}{ SSFF } & \multirow{6}{*}{ Present } & 15 & 3.403 & 17.316 & 19.388 & 38.319 & 51.039 \\
\hline & & 21 & 3.392 & 17.316 & 19.359 & 38.286 & 51.035 \\
\hline & & 27 & 3.385 & 17.316 & 19.341 & 38.266 & 51.035 \\
\hline & & 33 & 3.381 & 17.316 & 19.329 & 38.253 & 51.035 \\
\hline & & 37 & 3.379 & 17.316 & 19.324 & 38.247 & 51.035 \\
\hline & & 41 & 3.377 & 17.316 & 19.320 & 38.242 & 51.035 \\
\hline & New Ritz [5] & & 3.3670 & 17.316 & 19.293 & 38.211 & 51.035 \\
\hline & GDQM [22] & 15 & 2.549 & 17.316 & 17.662 & 36.576 & 51.039 \\
\hline \multirow{8}{*}{$\mathrm{CCFF}$} & \multirow{6}{*}{ Present } & 15 & 7.443 & 23.961 & 26.482 & 47.485 & 62.722 \\
\hline & & 21 & 7.280 & 23.900 & 26.525 & 47.431 & 62.707 \\
\hline & & 27 & 7.188 & 23.893 & 26.547 & 47.440 & 62.708 \\
\hline & & 33 & 7.125 & 23.894 & 26.564 & 47.463 & 62.709 \\
\hline & & 37 & 7.092 & 23.895 & 26.572 & 47.480 & 62.708 \\
\hline & & 41 & 7.065 & 23.896 & 26.580 & 47.496 & 62.708 \\
\hline & New Ritz [5] & & 6.919 & 23.904 & 26.585 & 47.651 & 62.706 \\
\hline & GDQM [22] & 15 & 7.873 & 23.615 & 23.873 & 44.587 & 62.730 \\
\hline \multirow{8}{*}{ CSFF } & \multirow{6}{*}{ Present } & 15 & 5.960 & 19.675 & 24.029 & 42.745 & 52.810 \\
\hline & & 21 & 5.767 & 19.525 & 24.190 & 42.714 & 52.802 \\
\hline & & 27 & 5.638 & 19.417 & 24.327 & 42.763 & 52.795 \\
\hline & & 33 & 5.550 & 19.338 & 24.425 & 42.819 & 52.787 \\
\hline & & 37 & 5.507 & 19.298 & 24.475 & 42.853 & 52.781 \\
\hline & & 41 & 5.472 & 19.265 & 24.514 & 42.884 & 52.776 \\
\hline & New Ritz [5] & & 5.351 & 19.075 & 24.671 & 43.088 & 52.707 \\
\hline & GDQM [22] & 15 & 5.780 & 20.703 & 20.926 & 40.296 & 52.255 \\
\hline \multirow{8}{*}{ SFFF } & \multirow{6}{*}{ Present } & 15 & 6.705 & 14.909 & 25.505 & 26.129 & 48.474 \\
\hline & & 21 & 6.691 & 14.908 & 25.476 & 26.077 & 48.463 \\
\hline & & 27 & 6.679 & 14.906 & 25.452 & 26.053 & 48.458 \\
\hline & & 33 & 6.671 & 14.905 & 25.435 & 26.040 & 48.456 \\
\hline & & 37 & 6.667 & 14.904 & 25.427 & 26.034 & 48.455 \\
\hline & & 41 & 6.664 & 14.904 & 25.421 & 26.029 & 48.454 \\
\hline & New Ritz [5] & & 6.6437 & 14.9015 & 25.3757 & 26.0005 & 48.4495 \\
\hline & GDQM [22] & 15 & 5.161 & 14.725 & 23.082 & 24.156 & 46.296 \\
\hline \multirow{8}{*}{ CFFF } & \multirow{6}{*}{ Present } & 15 & 3.893 & 9.687 & 20.934 & 27.780 & 30.032 \\
\hline & & 21 & 3.695 & 9.413 & 20.995 & 27.710 & 30.139 \\
\hline & & 27 & 3.583 & 9.192 & 21.067 & 27.635 & 30.311 \\
\hline & & 33 & 3.517 & 9.027 & 21.122 & 27.568 & 30.457 \\
\hline & & 37 & 3.487 & 8.942 & 21.151 & 27.529 & 30.536 \\
\hline & & 41 & 3.466 & 8.871 & 21.174 & 27.494 & 30.603 \\
\hline & New Ritz [5] & & 3.4712 & 8.5074 & 21.2864 & 27.1990 & 30.9590 \\
\hline & GDQM [22] & 15 & 3.898 & 9.459 & 20.206 & 26.150 & 26.500 \\
\hline \multirow{8}{*}{ FFFF } & \multirow{6}{*}{ Present } & 15 & 13.668 & 19.596 & 24.379 & 35.016 & 35.196 \\
\hline & & 21 & 13.616 & 19.596 & 24.342 & 34.978 & 35.037 \\
\hline & & 27 & 13.578 & 19.596 & 24.321 & 34.939 & 34.962 \\
\hline & & 33 & 13.553 & 19.596 & 24.308 & 34.909 & 34.920 \\
\hline & & 37 & 13.541 & 19.596 & 24.302 & 34.895 & 34.902 \\
\hline & & 41 & 13.532 & 19.596 & 24.298 & 34.883 & 34.887 \\
\hline & New Ritz [5] & & 13.4682 & 19.5961 & 24.2702 & 34.8009 & 34.8009 \\
\hline & GDQM [22] & 15 & 10.303 & 19.596 & 22.146 & 30.026 & 30.803 \\
\hline
\end{tabular}


Table 5. Percent error in solutions of two DQ approaches (present and GDQM) for natural frequencies of square plates with free corners (when non-uniform sampling points without adjacent $\delta$-points are used in the algorithms).

\begin{tabular}{|c|c|c|c|c|c|c|c|}
\hline Plate & Method & $n=m$ & $\Omega_{1}$ & $\Omega_{2}$ & $\Omega_{3}$ & $\Omega_{4}$ & $\Omega_{5}$ \\
\hline \multirow{2}{*}{$\mathrm{SSFF}$} & Present & 15 & 1.0685 & 0.0025 & 0.4942 & 0.2835 & 0.0085 \\
\hline & GDQM [22] & 15 & 24.2946 & 0 & 8.4538 & 4.2789 & 0.0078 \\
\hline \multirow{2}{*}{$\mathrm{CCFF}$} & Present & 15 & 7.5800 & 0.2397 & 0.3877 & 0.3480 & 0.0255 \\
\hline & GDQM [22] & 15 & 13.7881 & 1.2090 & 10.2012 & 6.4301 & 0.0383 \\
\hline \multirow{2}{*}{ CSFF } & Present & 15 & 11.3895 & 3.1438 & 2.6028 & 0.7957 & 0.1956 \\
\hline & GDQM [22] & 15 & 8.0172 & 8.5347 & 15.1798 & 6.4798 & 0.8576 \\
\hline \multirow{2}{*}{ SFFF } & Present & 15 & 0.9272 & 0.0519 & 0.5094 & 0.4930 & 0.0503 \\
\hline & GDQM [22] & 15 & 22.3174 & 1.1844 & 9.0390 & 7.0941 & 4.4448 \\
\hline \multirow{2}{*}{ CFFF } & Present & 15 & 12.1652 & 13.8716 & 1.6528 & 2.1369 & 2.9933 \\
\hline & GDQM [22] & 15 & 12.2955 & 11.1856 & 5.0755 & 3.8568 & 14.4029 \\
\hline \multirow{2}{*}{ FFFF } & Present & 15 & 1.4881 & 0.0005 & 0.4495 & 0.6194 & 1.1355 \\
\hline & GDQM [22] & 15 & 23.5013 & 0.0005 & 8.7523 & 13.7206 & 11.4879 \\
\hline
\end{tabular}
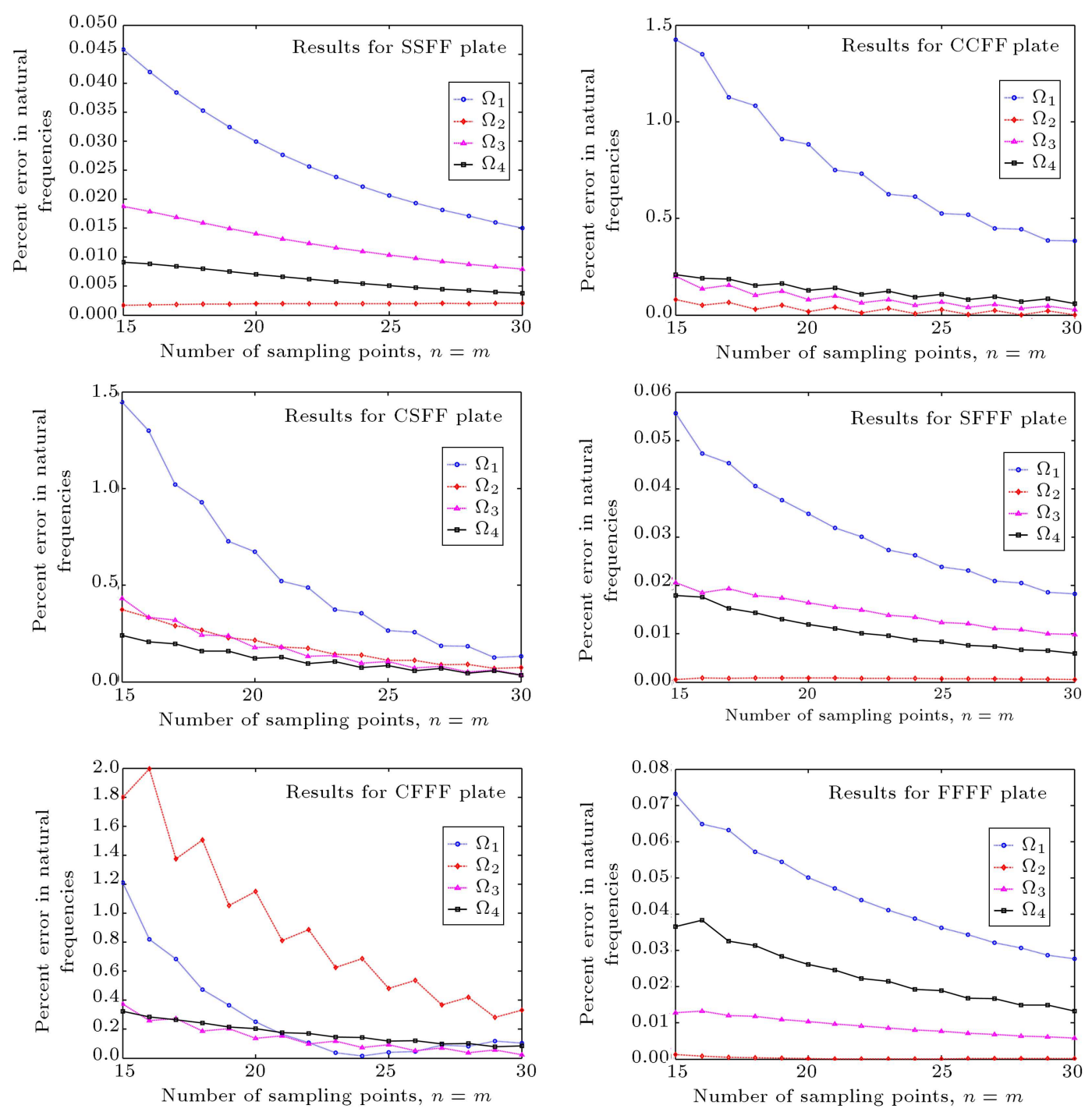

Figure 1. Convergence and accuracy of the first four natural frequencies of square plates involving free corners. 

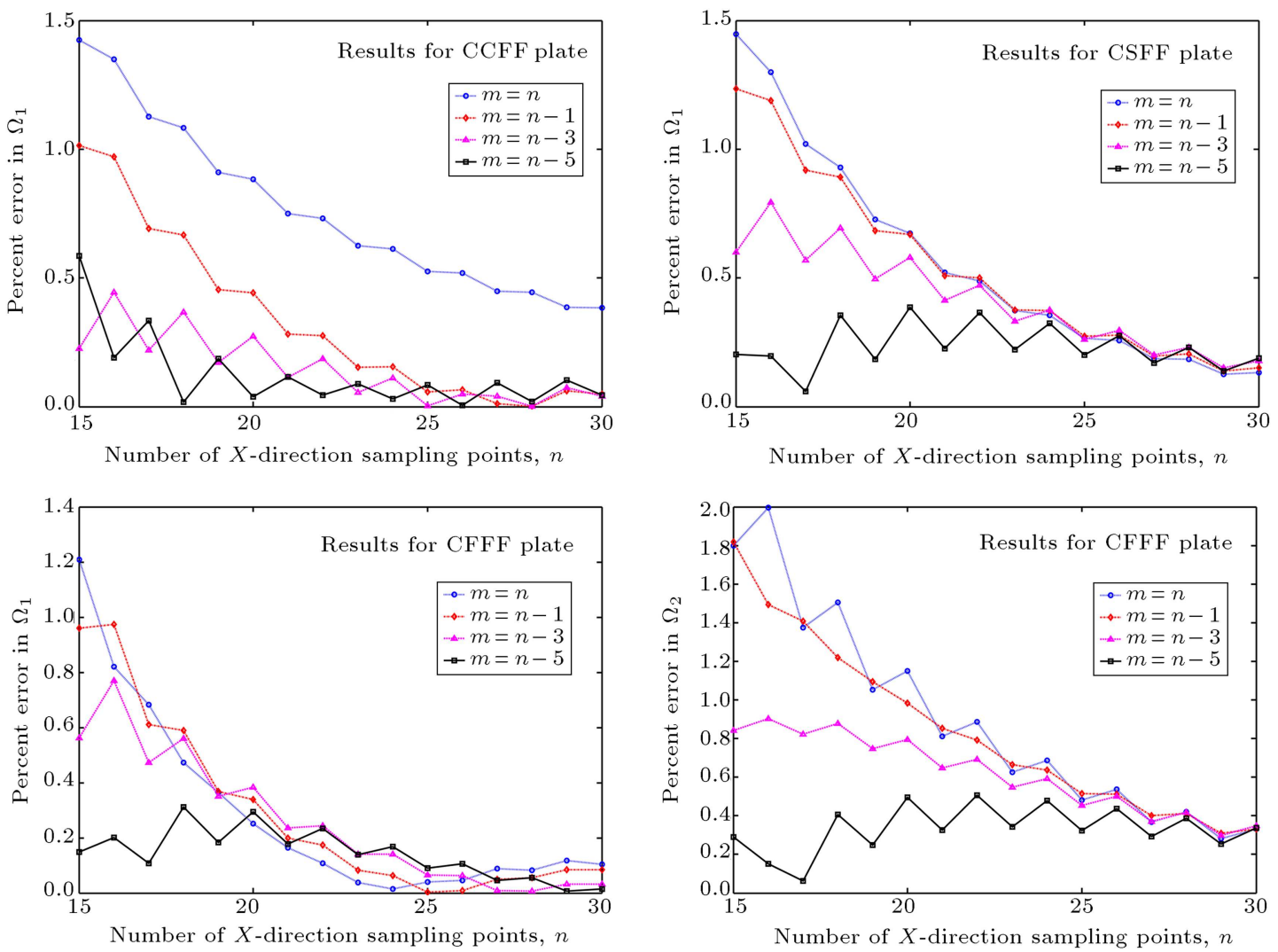

Figure 2. Convergence behavior and accuracy of natural frequencies of square plates with CCFF, CSFF, and CFFF boundary conditions.

difficulty is caused when an equal number of sampling points is considered in both coordinate directions of the plate. They have also shown that the convergence rate of the DQM is greatly enhanced by selecting an unequal number of sampling points in each coordinate direction of the plate. Our numerical experiments also confirmed this numerical observation for rectangular plates involving free corners and showed that, depending on the boundary conditions of the plate, the accuracy of numerical results could be improved when $n$ value was considered to be smaller or larger than $m$ value. Figure 2 presents the numerical results for plates with CCFF, CSFF, and CFFF boundary conditions. It can be seen that better accuracy and convergence rate can be achieved when $n$ value is chosen to be larger than $m$ value. The numerical results for plates with SSFF, SFFF and FFFF boundary conditions are shown in Figure 3 . It can be seen that in these cases, better accuracy and convergence rate are achieved by selecting $n$ value smaller than $m$ value.

As pointed out earlier in introduction, Shu and Du [22] reported that the solutions of the GDQM are very sensitive to the sampling point distributions when the plate under investigation involves some free corners. For instance, their procedure led to erroneous results when using the following type of sampling points:

$$
\begin{aligned}
& X_{i}=\frac{1}{2}\left[1-\cos \left(\frac{(i-1) \pi}{n-1}\right)\right], \quad i=1,2, \cdots, n, \\
& Y_{i}=\frac{1}{2}\left[1-\cos \left(\frac{(i-1) \pi}{m-1}\right)\right], \quad i=1,2, \cdots, m .
\end{aligned}
$$

To investigate the effect of sampling point distribution, we also solved the present problem using the above type of sampling points. Table 4 shows the convergence of solutions for the first five natural frequencies of square plates involving free corners when the coordinates of the sampling points are computed from Eqs. (56) and (57). The GDQM solution results of Shu and Du [22] are also shown for comparison purposes. Similar to Shu and Du [22], we considered an equal number of sampling points in both coordinate directions of the plate $(n=m)$. It can be seen that the results of present method show a monotonic convergence with respect to the number of sampling points while the numerical results of Shu and Du [22] do not exhibit any convergence trend for these cases. This implies that the solutions of the proposed method for plates involving free corners are not highly sensitive to the sampling point distribution. Table 5 presents a fair comparison of the present DQM and the GDQM when a mesh size of $15 \times 15$ is used. Note that 

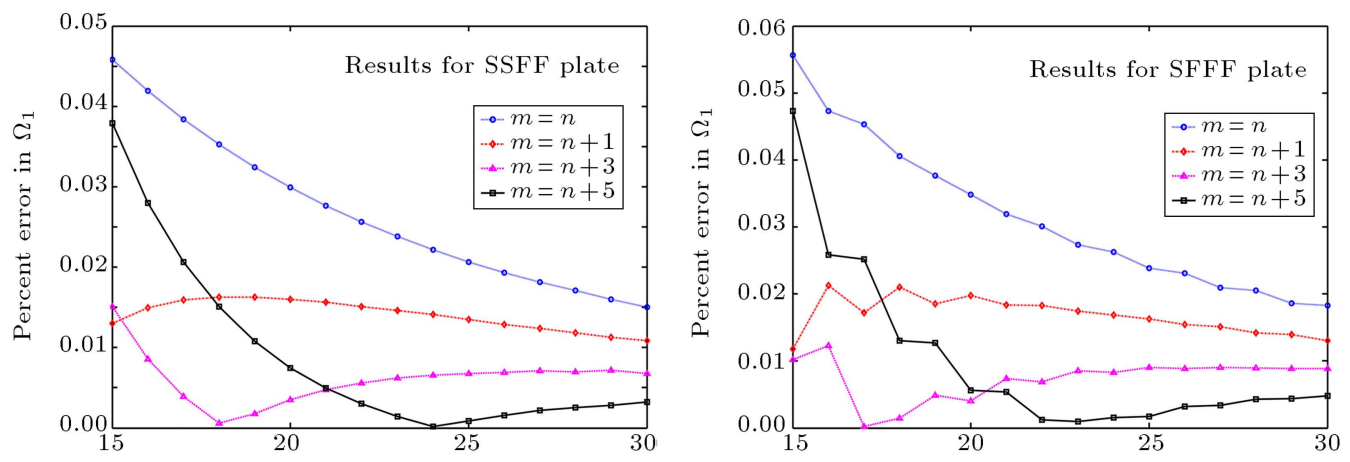

Number of $X$-direction sampling points, $n$
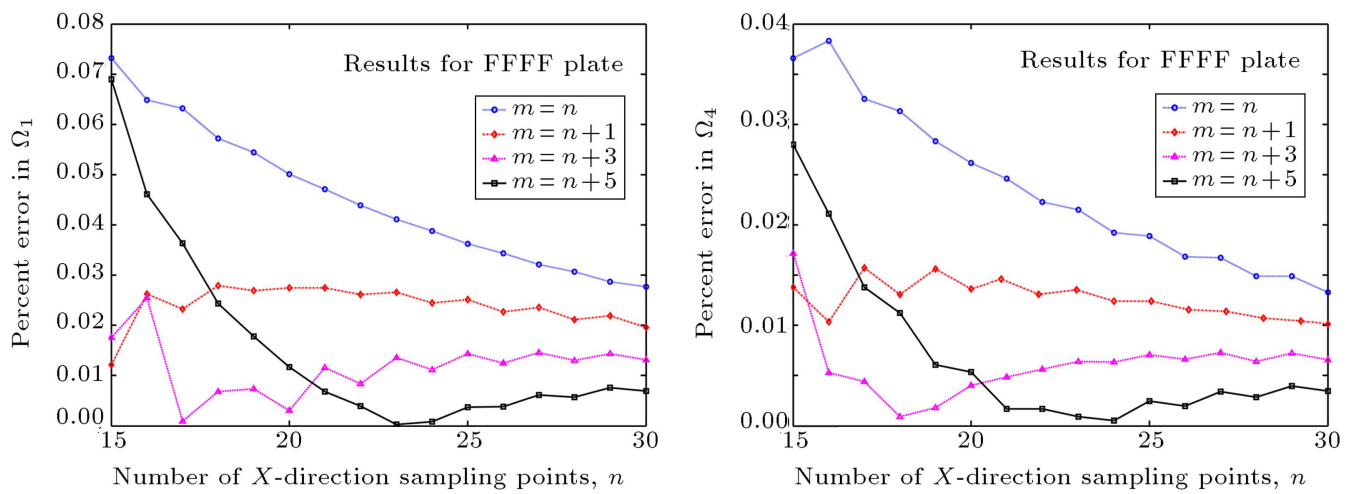

Figure 3. Convergence behavior and accuracy of natural frequencies of square plates with SSFF, SFFF, and FFFF boundary conditions.

the percent error in quadrature solutions (defined as $\left.\left|\Omega_{\mathrm{DQM}}-\Omega_{\mathrm{Ritz} \text { [5] }}\right| / \Omega_{\mathrm{Ritz}[5]} \times 100\right)$ is shown in this table. Needless to say that better accuracy is achieved by the proposed DQM.

\section{Conclusions}

A simple and accurate differential quadrature formulation is developed to study the free vibration of rectangular plates. The proposed formulation reduces the original plate problem to two simple beam problems whose solution procedure is significantly simpler than the case where the conventional DQM is fully applied to the plate problem. A simple scheme is also proposed to implement the free edge and free corner boundary conditions of the plate problem. It is revealed that the proposed method can produce much better accuracy than the GDQM for plates involving free corners. Unlike the GDQM, the solutions of the proposed method for plates with free corners are not very sensitive to the sampling point distribution.

\section{References}

1. Leissa, A.W. "The free vibration of rectangular plates", J. Sound Vib., 31(3), pp. 257-293 (1973).

2. Xiang, Y., Zhao, Y.B. and Wei, G.W. "Levy solutions for vibration of multi-span rectangular plates", Int. J. Mech. Sci., 44(6), pp. 1195-1218 (2002).
3. Bassily, S.F. and Dickinson, S.M. "On the use of beam functions for problems of plates involving free edges", ASME J. Appl. Mech., 42, pp. 858-864 (1975).

4. Bhat, R.B. "Natural frequencies of rectangular plates using characteristic orthogonal polynomials in the Rayleigh-Ritz method", J. Sound Vib., 102(4), pp. 493-499 (1985).

5. Eftekhari, S.A. and Jafari, A.A. "A novel and accurate Ritz formulation for free vibration of rectangular and skew plates", ASME J. Appl. Mech., 79(6), p. 064504 (2012).

6. Chakraverty, S. and Behera, L. "Free vibration of rectangular nanoplates using Rayleigh-Ritz method", Physica E. Low Dimens. Syst. Nanostruct., 56, pp. 357-363 (2014).

7. Fallah, A., Aghdam, M.M. and Kargarnovin, M.H. "Free vibration analysis of moderately thick functionally graded plates on elastic foundation using the extended Kantorovich method", Arch. Appl. Mech., 83(2), pp. 177-191 (2013).

8. Valizadeh, N., Bui, T.Q., Vu, V.T., Thai, H.T. and Nguyen, M.N. "Isogeometric simulation for buckling, free and forced vibration of orthotropic plates", Int. J. Appl. Mech., 5(2), 1350017 (2013).

9. Pachenari, Z. and Attarnejad, R. "Free vibration of tapered Mindlin plates using basic displacement functions", Arab. J. Sci. Eng., 39, pp. 4433-4449 (2014).

10. Tsiatas, G.C. and Yiotis, A.J. "A BEM-based meshless solution to buckling and vibration problems of 
orthotropic plates", Eng. Anal. Bound. Elem., 37(3), pp. 579-584 (2013).

11. Ragb, O., Matbuly, M.S. and Nassar, M. "Analysis of composite plates using moving least squares differential quadrature method", Appl. Math. Comput., 238(1), pp. 225-236 (2014).

12. Lal, R. and Saini, R. "Buckling and vibration of nonhomogeneous rectangular plates subjected to linearly varying in-plane force", Shock Vib., 20(5), pp. 879-894 (2013).

13. Wilson, A.J. and Rajasekaran, S. "Elastic stability of all edges clamped stepped and stiffened rectangular plate under uni-axial, bi-axial and shearing forces", Meccanica, 48(10), pp. 2325-2337 (2013).

14. Wu, Z.-J., Li, F.-M. and Wang, Y.-Z. "Vibration band gap properties of periodic Mindlin plate structure using the spectral element method", Meccanica, 49(3), pp. 725-737 (2014)

15. Ng, C.H.W., Zhao, Y.B. and Wei, G.W. "Comparison of discrete singular convolution and generalized differential quadrature for the vibration analysis of rectangular plates", Comput. Meth. Appl. Mech. Eng., 193(23-26), pp. 2483-2506 (2004).

16. Wang, $\mathrm{X}$. and $\mathrm{Xu}, \mathrm{S}$. "Free vibration analysis of beams and rectangular plates with free edges by the discrete singular convolution", J. Sound Vib., 329(10), pp. 1780-1792 (2010).

17. Wang, X., Bert, C.W. and Striz, A.G. "Differential quadrature analysis of deflection, buckling, and free vibration of beams and rectangular plates", Comput. Struct., 48(3), pp. 473-479 (1993).

18. Striz, A.G., Wang, X. and Bert, C.W. "Harmonic differential quadrature method and applications to analysis of structural components", Acta Mech., 111(1-2), pp. 85-94 (1995).

19. Karami, G. and Malekzadeh, P. "Application of a new differential quadrature methodology for free vibration analysis of plates", Int. J. Numer. Meth. Eng., 56(6), pp. 847-868 (2003).

20. Malekzadeh, P., Karami, G. and Farid, M. "A semianalytical DQEM for free vibration analysis of thick plates with two opposite edges simply supported", Comput. Meth. Appl. Mech. Eng., 193(45-47), pp. 4781-4796 (2004).

21. Malik, M. and Bert, C.W. "Implementing multiple boundary conditions in the DQ solution of higher-order PDE's: application to free vibration of plates", Int. J. Numer. Meth. Eng., 39, pp. 1237-1258 (1996).

22. Shu, C. and Du, H. "A generalized approach for implementing general boundary conditions in the GDQ free vibration analyses of plates", Int. J. Solids Struct., 34, pp. 837-846 (1997).

23. Bert, C.W. and Malik, M. "Differential quadrature method in computational mechanics: A review", ASME J. Appl. Mech. Rev., 49, pp. 1-28 (1996).
24. Bellman, R.E. and Casti, J. "Differential quadrature and long term integrations", J. Math. Anal. Appl., 34, pp. 235-238 (1971).

25. Bellman, R.E., Kashef, B.G. and Casti, J. "Differential quadrature: a technique for the rapid solution of nonlinear partial differential equations", Comput. Phys., 10, pp. 40-52 (1972).

26. Shu, C., Differential Quadrature and its Application in Engineering, Springer Publication (2000).

27. Zong, Z. and Zhang, Y., Advanced Differential Quadrature Methods, Chapman \& Hall (2009).

28. Civalek, O. "Application of differential quadrature (DQ) and harmonic differential quadrature (HDQ) for buckling analysis of thin isotropic plates and elastic columns", Eng. Struct., 26(2), pp. 171-186 (2004).

29. Wu, W.X., Shu, C. and Wang, C.M. "Vibration analysis of arbitrarily shaped membranes using local radial basis function-based differential quadrature method", J. Sound Vib., 306, pp. 252 - 270 (2007).

30. Eftekhari, S.A. and Jafari, A.A. "Numerical simulation of chaotic dynamical systems by the method of differential quadrature", Sci. Iran. B, 19(5), pp. 1299-1315 (2012).

31. Eftekhari, S.A. and Jafari, A.A. "Numerical solution of general boundary layer problems by the method of differential quadrature", Sci. Iran. B, 20(4), pp. 12781301 (2013).

32. Khani, S., Tabandeh, N. and Ghomshei, M.M. "Natural frequency analysis of non-uniform smart beams with piezoelectric layers, using differential quadrature method", Compos. Part B Eng., 58, pp. 303-311 (2014).

33. Torabi, K., Afshari, H. and Haji Aboutalebi, F. "A DQEM for transverse vibration analysis of multiple cracked non-uniform Timoshenko beams with general boundary conditions", Comput. Math. Appl., 67(3), pp. 527-541 (2014).

34. Asadi, H. and Aghdam, M.M. "Large amplitude vibration and post-buckling analysis of variable crosssection composite beams on nonlinear elastic foundation", Int. J. Mech. Sci., 79, pp. 47-55 (2014).

35. Eftekhari, S.A. and Jafari, A.A. "High accuracy mixed finite element-differential quadrature method for free vibration of axially moving orthotropic plates loaded by linearly varying in-plane stresses", Sci. Iran. B, 21(6), pp. 1933-1954 (2014).

36. Niu, Y., Wang, Z. and Zhang, W. "Nonlinear thermal flutter analysis of supersonic composite laminated panels using differential quadrature method", Int. J. Str. Stab. Dyn., 14(7), 1450030 (2014).

37. Heydarpour, Y., Malekzadeh, P. and Aghdam, M.M. "Free vibration of functionally graded truncated conical shells under internal pressure", Meccanica, 49(2) pp. 267-282 (2014).

38. Ansari, R., Mohammadi, V., Faghih Shojaei, M., Gholami, R. and Sahmani, S. "Surface stress effect on 
the postbuckling and free vibrations of axisymmetric circular Mindlin nanoplates subject to various edge supports", Compos. Struct., 112, pp. 358-367 (2014).

39. Rajasekaran, S. and Tochaei, E.N. "Free vibration analysis of axially functionally graded tapered Timoshenko beams using differential transformation element method and differential quadrature element method of lowest-order", Meccanica, 49(4), pp. 9951009 (2014).

40. Fantuzzi, N., Tornabene, F., Viola, E. and Ferreira, A.J.M. "A strong formulation finite element method (SFEM) based on RBF and GDQ techniques for the static and dynamic analyses of laminated plates of arbitrary shape", Meccanica, 49(10), pp. 2503-2542 (2014).

41. Tornabene, F., Fantuzzi, N., Viola, E. and Carrera, E. "Static analysis of doubly-curved anisotropic shells and panels using CUF approach, differential geometry and differential quadrature method", Compos. Struct., 107, pp. 675-697 (2014).

42. Fantuzzi, N., Tornabene, F. and Viola, E. "Generalized differential quadrature finite element method for vibration analysis of arbitrarily shaped membranes", Int. J. Mech. Sci., 79, pp. 216-251 (2014).

43. Vosoughi, A.R., Banan, Mo.R., Banan, Ma.R. and Malekzadeh, P. "Hybrid FE-IDQ-CG method for dynamic parameters estimation of multilayered halfspace", Compos. Part B Eng., 56, pp. 74-82 (2014).

44. Golbahar Haghighi, M.R., Malekzadeh, P. and Afshari, M. "Inverse estimation of heat flux and pressure in functionally graded cylinders with finite length", Compos. Struct., 121, pp. 1-15 (2015).

45. Behera, L. and Chakraverty, S. "Application of differential quadrature method in free vibration analysis of nanobeams based on various nonlocal theories", Comput. Math. Appl., 69(12), pp. 1444-1462 (2015).

46. Eftekhari, S.A. "A differential quadrature procedure with regularization of the Dirac-delta function for numerical solution of moving load problem", Latin Am. J. Solids Struct., 12, pp. 1241-1265 (2015)

47. Eftekhari, S.A. "A modified differential quadrature procedure for numerical solution of moving load problem", J. Mech. Eng. Sci., 230(5), pp. 715-731 (2016).

48. Eftekhari, S.A. and Jafari, A.A. "A mixed modaldifferential quadrature method for free and forced vibration of beams in contact with fluid", Meccanica, 49, pp. 535-564 (2014).

49. Eftekhari, S.A. "Pressure-based and potential-based mixed Ritz-differential quadrature formulations for free and forced vibration of Timoshenko beams in contact with fluid", Meccanica, 51(1), pp. 179-210 (2015).

50. Quan, J.R. and Chang, C.T. "New insights in solving distributed system equations by the quadrature methods. Part I: analysis", Comput. Chem. Eng., 13, pp. 779-788 (1989).
51. Eftekhari, S.A. and Jafari, A.A. "A mixed method for forced vibration of multi-span rectangular plates carrying moving masses", Arab. J. Sci. Eng., 39, pp. 3225-3250 (2014).

52. Moler, C.B. and Stewart, G.W. "An algorithm for generalized matrix eigenvalue problems", SIAM J. Numer. Anal., 10(2), pp. 241-256 (1973).

53. Eftekhari, S.A. and Jafari, A.A. "High accuracy mixed finite element-Ritz formulation for free vibration analysis of plates with general boundary conditions", Appl. Math. Comput., 219, pp. 312-1344 (2012).

54. Bert, C.W. and Malik, M. "The differential quadrature method for irregular domains and application to plate vibration", Int. J. Mech. Sci., 38(6), pp. 589-606 (1996).

\section{Appendix A}

Elements of matrices $\left[\mathrm{A}^{(4)}\right]_{b l}$ and $\left[\mathrm{A}^{(2)}\right]_{b l}$ The elements of matrices $\left[\mathbf{A}^{(4)}\right]_{b l}$ and $\left[\mathbf{A}^{(2)}\right]_{b l}$ depend on the boundary conditions of the plate in the $X$ direction and can be obtained from quadrature analog equations given in Section 4.1 .2 as follows:

(I) Simply supported end condition at $X=X_{1}$ :

$$
\begin{aligned}
{\left[\mathbf{A}^{(4)}\right]_{b l} } & =\left[\begin{array}{ccccc}
1 & 0 & 0 & \cdots & 0 \\
A_{11}^{(2)} & A_{12}^{(2)} & A_{13}^{(2)} & \cdots & A_{1 n}^{(2)}
\end{array}\right](\mathrm{A} .1) \\
{\left[\mathbf{A}^{(2)}\right]_{b l} } & =\left[\begin{array}{cccc}
0 & 0 & \cdots & 0 \\
0 & 0 & \cdots & 0
\end{array}\right] .
\end{aligned}
$$

(II) Clamped end condition at $X=X_{1}$ :

$$
\begin{aligned}
{\left[\mathbf{A}^{(4)}\right]_{b l} } & =\left[\begin{array}{ccccc}
1 & 0 & 0 & \cdots & 0 \\
A_{11}^{(1)} & A_{12}^{(1)} & A_{13}^{(1)} & \cdots & A_{1 n}^{(1)}
\end{array}\right] \\
{\left[\mathbf{A}^{(2)}\right]_{b l} } & =\left[\begin{array}{llll}
0 & 0 & \cdots & 0 \\
0 & 0 & \cdots & 0
\end{array}\right] .
\end{aligned}
$$

(III) Free end condition at $X=X_{1}$ :

$$
\begin{aligned}
& {\left[\mathbf{A}^{(4)}\right]_{b l}=\left[\begin{array}{llll}
A_{11}^{(2)} & A_{12}^{(2)} & \cdots & A_{1 n}^{(2)} \\
A_{11}^{(3)} & A_{12}^{(3)} & \cdots & A_{1 n}^{(3)}
\end{array}\right],} \\
& {\left[\mathbf{A}^{(2)}\right]_{b l}=\frac{1}{2}\left[\begin{array}{cc}
\mu & 0 \\
(2-\mu) A_{11}^{(1)} & (2-\mu) A_{12}^{(1)}
\end{array}\right.} \\
& \left.\begin{array}{ccc}
0 & \cdots & 0 \\
(2-\mu) A_{13}^{(1)} & \cdots & (2-\mu) A_{1 n}^{(1)}
\end{array}\right] \text { (A. }
\end{aligned}
$$




\section{Appendix B}

Elements of matrices $\left[\mathrm{A}^{(4)}\right]_{b r}$ and $\left[\mathrm{A}^{(2)}\right]_{b r}$

The elements of matrices $\left[\mathbf{A}^{(4)}\right]_{b r}$ and $\left[\mathbf{A}^{(2)}\right]_{b r}$ can also be determined from quadrature analog equations given in Section 4.1.2 as follows:

(I) Simply supported end condition at $X=X_{n}$ :

$$
\begin{aligned}
{\left[\mathbf{A}^{(4)}\right]_{b r} } & =\left[\begin{array}{ccccc}
A_{n 1}^{(2)} & A_{n 2}^{(2)} & \cdots & A_{n(n-1)}^{(2)} & A_{n n}^{(2)} \\
0 & 0 & \cdots & 0 & 1
\end{array}\right] \\
{\left[\mathbf{A}^{(2)}\right]_{b r} } & =\left[\begin{array}{llll}
0 & 0 & \cdots & 0 \\
0 & 0 & \cdots & 0
\end{array}\right] .
\end{aligned}
$$

(II) Clamped end condition at $X=X_{n}$ :

$$
\begin{aligned}
& {\left[\mathbf{A}^{(4)}\right]_{b r}=\left[\begin{array}{ccccc}
A_{n 1}^{(1)} & A_{n 2}^{(1)} & \cdots & A_{n(n-1)}^{(1)} & A_{n n}^{(1)} \\
0 & 0 & \cdots & 0 & 1
\end{array}\right]} \\
& {\left[\mathbf{A}^{(2)}\right]_{b r}=\left[\begin{array}{llll}
0 & 0 & \cdots & 0 \\
0 & 0 & \cdots & 0
\end{array}\right] .}
\end{aligned}
$$

(III) Free end condition at $X=X_{n}$ :

$$
\begin{aligned}
& {\left[\mathbf{A}^{(4)}\right]_{b r}=\left[\begin{array}{llll}
A_{n 1}^{(3)} & A_{n 2}^{(3)} & \cdots & A_{n n}^{(3)} \\
& & & \\
A_{n 1}^{(2)} & A_{n 2}^{(2)} & \cdots & A_{n n}^{(2)}
\end{array}\right]} \\
& {\left[\mathbf{A}^{(2)}\right]_{b r}=\frac{1}{2}\left[\begin{array}{ccc}
(2-\mu) A_{n 1}^{(1)} & (2-\mu) A_{n 2}^{(1)} & \ldots \\
0 & 0 & \cdots
\end{array}\right.} \\
& \left.\begin{array}{cc}
(2-\mu) A_{n(n-1)}^{(1)} & (2-\mu) A_{n n}^{(1)} \\
0 & \mu
\end{array}\right]
\end{aligned}
$$

\section{Appendix C}

Elements of matrix $\left[\tilde{\mathbf{K}}_{b l}\right]$

The elements of matrix $\left[\tilde{\mathbf{K}}_{b l}\right]$ depend on the boundary conditions of the plate in $Y$-direction and can be obtained from quadrature analog equations given in Section 4.2.2 as follows:

(I) Simply supported end condition at $Y=Y_{1}$ :

$$
\begin{aligned}
& {\left[\tilde{\mathbf{K}}_{b l}\right]=\left[\begin{array}{cc}
{[\hat{\mathbf{I}}]} & {[\mathbf{0}]} \\
B_{11}^{(2)}[\hat{\mathbf{I}}] & B_{12}^{(2)}[\hat{\mathbf{I}}]
\end{array}\right.} \\
& \left.\begin{array}{ccc}
{[\mathbf{0}]} & \cdots & {[\mathbf{0}]} \\
B_{13}^{(2)}[\hat{\mathbf{I}}] & \cdots & B_{1 m}^{(2)}[\hat{\mathbf{I}}]
\end{array}\right] .
\end{aligned}
$$

(II) Clamped end condition at $Y=Y_{1}$ :

$$
\begin{aligned}
& {\left[\tilde{\mathbf{K}}_{b l}\right]=\left[\begin{array}{cc}
{[\hat{\mathbf{I}}]} & {[0]} \\
B_{11}^{(1)}[\hat{\mathbf{I}}] & B_{12}^{(1)}[\hat{\mathbf{I}}]
\end{array}\right.} \\
& \left.\begin{array}{ccc}
{[\mathbf{0}]} & \cdots & {[\mathbf{0}]} \\
B_{13}^{(1)}[\hat{\mathbf{I}}] & \cdots & B_{1 m}^{(1)}[\hat{\mathbf{I}}]
\end{array}\right] .
\end{aligned}
$$

(III) Free end condition at $Y=Y_{1}$ :

$$
\begin{aligned}
& {\left[\tilde{\mathbf{K}}_{b l}\right]=\left[\begin{array}{llll}
B_{11}^{(2)}[\hat{\mathbf{I}}] & B_{12}^{(2)}[\hat{\mathbf{I}}] & \cdots & B_{1 m}^{(2)}[\hat{\mathbf{I}}] \\
B_{11}^{(3)}[\hat{\mathbf{I}}] & B_{12}^{(3)}[\hat{\mathbf{I}}] & \cdots & B_{1 m}^{(3)}[\hat{\mathbf{I}}]
\end{array}\right]} \\
& +\frac{1}{\lambda^{2}}\left[\begin{array}{cc}
\mu\left[\tilde{\mathbf{A}}^{(2)}\right] & {[\mathbf{0}]} \\
(2-\mu) B_{11}^{(1)}\left[\tilde{\mathbf{A}}^{(2)}\right] & (2-\mu) B_{12}^{(1)}\left[\tilde{\mathbf{A}}^{(2)}\right]
\end{array}\right. \\
& {[0] \quad \ldots \quad[0]} \\
& \left.(2-\mu) B_{13}^{(1)}\left[\tilde{\mathbf{A}}^{(2)}\right] \cdots(2-\mu) B_{1 m}^{(1)}\left[\tilde{\mathbf{A}}^{(2)}\right]\right],
\end{aligned}
$$

where $\left[\tilde{\mathbf{A}}^{(2)}\right]$ has already been defined in Eqs. (43)-(46), $[\hat{\mathbf{I}}]$ and $[\mathbf{0}]$ are identity and zero matrices of order $n_{f} \times$ $n_{f}\left(n_{f}=n\right)$.

\section{Appendix D}

\section{Elements of matrix $\left[\tilde{\mathbf{K}}_{b r}\right]$}

The elements of matrix $\left[\tilde{\mathbf{K}}_{b r}\right]$ can also be determined from quadrature analog equations given in Section 3.1 as follows:

(I) Simply supported end condition at $Y=Y_{m}$ :

$$
\begin{array}{r}
{\left[\tilde{\mathbf{K}}_{b r}\right]=\left[\begin{array}{ccc}
B_{m 1}^{(2)}[\hat{\mathbf{I}}] & B_{m 2}^{(2)}[\hat{\mathbf{I}}] & \cdots \\
{[\mathbf{0}]} & {[\mathbf{0}]} & \cdots \\
& B_{m(m-1)}^{(2)}[\hat{\mathbf{I}}] & B_{m m}^{(2)}[\hat{\mathbf{I}}] \\
{[\mathbf{0}]} & {[\hat{\mathbf{I}}]}
\end{array}\right] .}
\end{array}
$$

(II) Clamped end condition at $Y=Y_{m}$ :

$$
\begin{array}{r}
{\left[\tilde{\mathbf{K}}_{b r}\right]=\left[\begin{array}{ccc}
B_{m 1}^{(1)}[\hat{\mathbf{I}}] & B_{m 2}^{(1)}[\hat{\mathbf{I}}] & \cdots \\
{[\mathbf{0}]} & {[\mathbf{0}]} & \cdots \\
B_{m(m-1)}^{(1)}[\hat{\mathbf{I}}] & \left.B_{m m}^{(1)}[\hat{\mathbf{I}}]\right] \\
{[\mathbf{0}]} & {[\hat{\mathbf{I}}]}
\end{array}\right]}
\end{array}
$$


(III) Free end condition at $Y=Y_{m}$

$$
\begin{aligned}
& {\left[\tilde{\mathbf{K}}_{b r}\right]=\left[\begin{array}{llll}
B_{m 1}^{(3)}[\hat{\mathbf{I}}] & B_{m 2}^{(3)}[\hat{\mathbf{I}}] & \cdots & B_{m m}^{(3)}[\hat{\mathbf{I}}] \\
B_{m 1}^{(2)}[\hat{\mathbf{I}}] & B_{m 2}^{(2)}[\hat{\mathbf{I}}] & \cdots & B_{m m}^{(2)}[\hat{\mathbf{I}}]
\end{array}\right]} \\
& +\frac{1}{\lambda^{2}}\left[\begin{array}{cc}
(2-\mu) B_{m 1}^{(1)}\left[\tilde{\mathbf{A}}^{(2)}\right] & (2-\mu) B_{m 2}^{(1)}\left[\tilde{\mathbf{A}}^{(2)}\right] \\
{[\mathbf{0}]} & {[\mathbf{0}]}
\end{array}\right. \\
& \cdots(2-\mu) B_{m(m-1)}^{(1)}\left[\tilde{\mathbf{A}}^{(2)}\right](2-\mu) B_{m m}^{(1)}\left[\tilde{\mathbf{A}}^{(2)}\right] \\
& \left.\cdots \quad[\mathbf{0}] \quad \mu\left[\tilde{\mathbf{A}}^{(2)}\right] \quad\right](\mathrm{D} .3)
\end{aligned}
$$

where the matrices $\left[\tilde{\mathbf{A}}^{(2)}\right],[\hat{\mathbf{I}}]$, and $[\mathbf{0}]$ have already been defined in Appendix C.

\section{Biographies}

Seyyed Aboozar Eftekhari was born in Karaj, Iran, in 1980. He received a BS degree in Mechanical Engineering from Sharif University of Technology, Tehran,
Iran, in 2003, an MS degree in Mechanical Engineering from Shiraz University, Shiraz, Iran, in 2006, and a $\mathrm{PhD}$ degree in Mechanical Engineering from K. N. Toosi University of Technology, Tehran, Iran, in 2013.

He is currently a member of Young Researchers and Elite Club, Karaj Branch, Islamic Azad University, Karaj, Iran. His research interests include applied mathematics, vibration of continuous systems, and fluid-structure interaction. He has published over 20 research papers on related subjects, including the study of the behavior of beams and plates under moving loads, mathematical modeling of vibration problem of beams and plates, and numerical solution of nonlinear differential equations in engineering and applied sciences.

Ali Asghar Jafari received his $\mathrm{PhD}$ degree in Mechanical Engineering from University of Wollongong, Australia, in 1994. He is currently a Full Professor in the Department of Mechanical Engineering at K. N. Toosi University of Technology, Iran. His research fields are dynamics and vibrations of beams, plates and shells, composite structures, and automotive engineering. 